Memoirs of the College of Science, University of Kyoto, Series A

Vol. XXXIII, Mathematics No. 1, 1960.

\title{
A global foundation of Finsler geometry
}

\author{
By \\ Makoto MATSumoto \\ (Received April 15, 1960)
}

\section{Introduction}

The purpose of the present paper is to give a global foundation of connections in Finsler spaces by means of the general theory of connections in differentiable fibre bundles.

Since the notion of connections in fibre bundles has been given a modern formulation, many authors have tried to establish the theory of Finsler geometry from this new point of view. Recently, T. Ötsuki $[8 ; 9 ; 10]$ dealed with our subject in detail, and his treatment seems to be rather complicated. Besides, M. Hashiguchi [4] discussed the parallel displacements and showed that the euclidean connection determined by E. Cartan [2] is the shortest and the fittest from a natural standpoint. Further, L. Auslander [1] generalised to Finsler geometry some global theorems concerning positive curvature. His starting point was also the connection for a Finsler manifold as calculated by E. Cartan and used the equations of structure which were given by S. S. Chern [3]. This theory of connections in a Finsler space given by S. S. Chern was formulated rigorously by S. Kashiwabara [5] in the viewpoint of theory of connections in fibre bundles.

Roughly speaking, the euclidean connection considered by $\mathrm{E}$. Cartan is the one in the principal bundle over the line bundle of a given manifold. If we try to define such a connection in a general differentiable fibre bundle, it is quite natural to pay attention to the centre $Z$ of the structural Lie group $G$. Because the centre of the general linear group $G L(n, R)$ is the set of matrices, which are of the form $\left(a \delta_{j}^{j}\right)$, where $a \in R-0$, and $\delta_{j}^{i}(i, j=1, \cdots, n)$ are the Kronecker's deltas. In this point of view, we shall define, in Chapters I and II, a general Finsler bundle $\mathfrak{Q}$ and a Finsler 
connection, which is invariant under central transformation. That is, given a differentiable fibre bundle $\mathfrak{B}$ over a differentiable manifold $M$, we consider the associated principal bundle $\mathfrak{r}_{3}$. Then, the Finsler bundle $\cong$ is defined as the principal bundle over the bundle space $B$ of $\mathfrak{B}$ induced from $\mathfrak{F}$. The central transformation of the bundle space $Q$ of $\mathfrak{Z}$ by an element of $Z$ will be defined in $\S 2$. In Chapters III and IV, we shall develop the theory of linear Finsler connection, where the original fibre bundle $\mathfrak{B}$ is the tangent bundle of $M$. In the last section, we shall devote ourself to the study of the euclidean connection determined by E. Cartan.

In conclusion, I wish to express my sincere gratitude to Dr. J. Kanitani for his continued encouragement. I have also had invaluable assistance and criticism by Dr. S. Takizawa.

\section{Chapter I. Finsler bundles and trivial connections}

\section{$\S 1$. Differentiable fibre bundles $\cong$ and $\tilde{\mathfrak{B}}$}

We consider a differentiable manifold $M$ of dimension $n$. By differentiability we shall always understand that of class $C^{\infty}$. We denote by $\mathfrak{B}=\{B, \tau, M, V, G\}$ a differentiable fibre bundle over the base space $M$. The differentiable mapping $\tau$ of the bundle space $B$ onto $M$ is the projection of $\mathfrak{B}$. The structural group $G$ is the Lie group which acts differentiably on the standard fibre $V$ to the left.

Let $\mathfrak{S}_{\beta}=\{P, \rho, M, G, G\}$ be the principal bundle associated with $\mathfrak{B}[12$, p. 35]. The mapping $\rho$ is the projection $P \rightarrow M$. Each point $p \in P$ may be considered as an admissible mapping of $V$ onto the fibre $V(x)$ over the point $x=\rho(p) \in M$. We denote by $R_{g}$ the right translation of $P$ by an element $g \in G$. Let $\left\{U_{\alpha}\right\}$ be an open covering of $M$ by coordinate neighborhoods and $\chi_{a}: U_{a} \times G \rightarrow \rho^{-1}\left(U_{a}\right)$ the coordinate functions corresponding to $U_{a}$. Then the right translation $R_{g}$ is expressed by

$$
R_{g}(p)=p \cdot g=\chi_{\omega}\left(x, \quad \chi_{\alpha, x}^{-1}(p) \cdot g\right), \quad x=\rho(p) \in U_{\alpha} .
$$

Next, we construct the principal bundle $\tau^{-1}(\mathfrak{F})$ induced from $\mathfrak{F}$ by the projection $\tau: B \rightarrow M$ of $\mathfrak{B}$, and we denote $\tau^{-1}(\mathfrak{B})$ by $\mathfrak{Q}=\{Q, \sigma, B, G, G\},[12$, p. 47]. The bundle space $Q$ is the submanifold of the product $B \times P$ and is defined by

$$
Q=\{(b, p) \in B \times P: \tau(b)=\rho(p)\} .
$$

The projection $\sigma$ of $\mathscr{Q}$ is the canonical mapping $Q \rightarrow B$, such that 
$\sigma((b, p))=b$. Let $R_{g}^{\prime}$ be the right translation of $Q$ by an element $g \in G$. If we take an open set $V_{a}=\tau^{-1}\left(U_{a}\right)$, then $\left\{V_{a}\right\}$ is an open covering of $B$ considered as the base space of $\mathfrak{Q}$, and coordinate functions $\psi_{\infty}$ corresponding to $V_{a}$ are given by

$$
\psi_{\infty}(b, g)=\left(b, \quad \chi_{a}(\tau(b), g)\right), \quad b \in V_{a}, g \in G,
$$

where $\chi_{a}$ are the coordinate functions of $\Re$. Since a right translation $R_{g}^{\prime}$ of $Q$ is expressed by the similar equation to the one of a right translation $R_{g}$ of $P$ in terms of $\psi_{a}$, then we obtain immediately

$$
q \cdot g=(\sigma(q), \eta(q) \cdot g), \quad q \in Q, g \in G,
$$

where $\eta$ is the canonical mapping $Q \rightarrow P$, such that $\eta((b, p))=p$.

We consider next the fibre bundle $\tilde{\mathfrak{B}}=\{\widetilde{B}, \tilde{\sigma}, B, V, G\}$ over the manifold $B$, which is associated with $\mathfrak{Q}$ and has $V$ as the standard fibre. In order to construct the bundle space $\widetilde{B}$, we introduce an equivalence relation in the product $Q \times V$ as follows : $(q, v) \in Q \times V$ is equivalent to $\left(q^{\prime}, v^{\prime}\right) \in Q \times V$ if and only if there exists an element $g \in G$ such that $q^{\prime}=q \cdot g$ and $v^{\prime}=g^{-1} \cdot v$. We define now the space $\widetilde{B}$ as the quotient space of $Q \times V$ by the above equivalence relation, and write simply by $q v$ the equivalence class of $(q, v)$. The projection $\tilde{\sigma}$ of $\tilde{\mathfrak{B}}$ is given by $\tilde{\sigma}(q v)=\sigma(q)$. Each point $q \in Q$ is considered as an admissible mapping of $V$ onto the fibre $V(b)$ of $\tilde{\mathfrak{B}}$ over the point $b=\sigma(q) \in B$, such that $q(v)=q v, v \in V$.

Now, we shall show that the bundle space $\widetilde{B}$ may be identified with $\bigcup_{x \in M} V(x) \times V(x)$, where $V(x)$ is a fibre over a point $x \in M$ of the original bundle $\mathfrak{B}$. In order to prove this, we shall introduce a mapping $i: \widetilde{B} \rightarrow B \times B$, such that

$$
i(q v)=(\sigma(q),(\eta(q))(v)), \quad q v \in \widetilde{B} .
$$

If we put $q=(b, p)$, then the mapping $i$ is expressed simply in the form $i((b, p) v)=(b, p(v))$. Since $\tau(b)=\rho(p)$, the mapping $i$ transforms $\widetilde{B}$ into $\bigcup_{x \in M} V(x) \times V(x)$. It is easily seen that $i$ as thus defined is one-to-one, and hence the above statement is established. On using the identification $i$, the projection $\tilde{\sigma}$ is rewritten in the form $\tilde{\sigma}(q v)=b$, where $q v=\left(b, b^{\prime}\right), b, b^{\prime} \in V(x), x=\tau(b)=\tau\left(b^{\prime}\right) \in M$.

\section{§ 2. Finsler fibre bundles}

We shall define, in this section, central transformations $C_{z}$ and 
$C_{z}^{\prime}$ of the manifolds $B$ and $Q$ respectively, which will be important in the following. Let $Z$ be the centre of the structural Lie group $G$ of the original bundle $\mathfrak{B}$. A central transformation $C_{z}$ of the bundle space $B$ of $\mathfrak{B}$ by an element $z \in Z$ is given by

$$
C_{z}(b)=z \cdot b=p\left(z \cdot p^{-1}(b)\right), \quad p \in \rho^{-1} \circ \tau(b),
$$

where $p \in P$ is to be considered as an admissible mapping of $\mathfrak{B}$. If we take account of the fact that $z \in Z$ commutes with any $g \in G$, then we see that the definition of $C_{z}$ is independent of the choice of $p \in \rho^{-1} \circ \tau(b)$. It is clear that $C_{z}$ acts on each fibre and gives an equivalence relation in $B$. Therefore, if we denote by $B^{*}$ the quotient space of $B$ by central transformations, then we have naturally a fibre bundle $\mathfrak{B}^{*}=\left\{B^{*}, \tau^{*}, M, V^{*}, G / Z\right\}$, where the projection $\tau^{*}$ is the mapping induced from $\tau$, and the standard fibre $V^{*}$ is the quotient space of $V$ by $Z$.

The central transformation $C_{z}$ induces naturally a transformation $C_{z}^{\prime}$ of the bundle space $Q$ of $\mathfrak{Q}$ over $B$, which is given by

$$
C_{z}^{\prime}(q)=z \cdot q=(z \cdot \sigma(q), \eta(q)), \quad q \in Q .
$$

This is called also the central transformation of $Q$ by an element $z \in Z$. From the relation $\sigma \circ C_{z}^{\prime}=C_{z} \circ \sigma$, it follows that $C_{z}^{\prime}$ is a map-

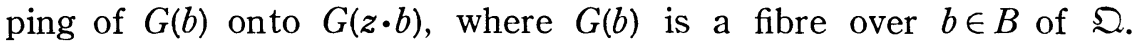
Furthermore, we see easily that $C_{z}^{\prime}$ commutes with a right translation $R_{g}^{\prime}$ of $Q$.

In the first section, we defined the principal bundle $\mathfrak{Q}$ as the induced bundle $\tau^{-1}(\mathfrak{S})$. Similarly, we can introduce an induced bundle $\tau^{*-1}\left(\mathfrak{A}^{*}\right)$ from $\boldsymbol{F}^{*}$ by the projection $\tau^{*}: B^{*} \rightarrow M$, and denote it by $\mathfrak{\Omega}^{*}=\left\{Q^{*}, \sigma^{*}, B^{*}, G, G\right\}$. We write by $b^{*}$ the equivalence class of $b \in B$ given by central transformations, and define the mapping

$$
r: B \rightarrow B^{*}, \quad r(b)=b^{*}, b \in B .
$$

Let $\bar{r}: Q \rightarrow Q^{*}$ be the induced mapping from $r$, such that $\bar{r}(q)=$ $(r \circ \sigma(q), \eta(q)), q \in Q$. We can easily prove that the mapping $\bar{r}$ gives the bundle mapping $\mathfrak{\Omega} \rightarrow \mathfrak{\Omega}$, such that all of the mapping transformations are equal to the identity [12, p. 9]. The induced bundle $\mathfrak{2 *}$ will be called the Finsler fibre bundle of the manifold $M$ constructed from the fibre bundle $\mathfrak{B}$ over $M$.

We constructed, in $\S 1$, the associated bundle $\tilde{\mathfrak{B}}$ with $\Omega$. We have now similarly the fibre bundle $\tilde{\mathfrak{B}}^{*}=\left\{\widetilde{B}^{*}, \tilde{\sigma}^{*}, B^{*}, V, G\right\}$ associated with the Finsler bundle $\mathfrak{}$ *. In this case also, a point 
of the bundle space $\widetilde{B}^{*}$ is identified with a pair $\left(b^{*}, b^{\prime}\right), b^{*} \in B^{*}$, $b^{\prime} \in B$, such that $\tau^{*}\left(b^{*}\right)=\tau\left(b^{\prime}\right)$. Then the projection $\tilde{\sigma}^{*}$ is given by $\tilde{\sigma}^{*}\left(\left(b^{*}, b^{\prime}\right)\right)=b^{*}$, and $b^{*}$ is called the element of support of $b^{\prime}[2$, p. 4$]$.

\section{$\S 3$. Trivial connections in the principal bundle $\cong$}

Let $\mathfrak{F}=\{P, \rho, M, G, G\}$ be the principal bundle as mentioned

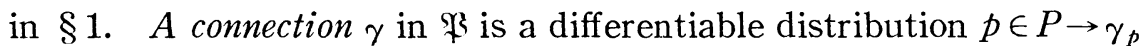
(=subspace of the tangent space $P_{p}$ at $p$ of $P$ ) which satisfies the following two conditions $[7$, p. 25$]$ :

1) The tangent space $P_{p}$ at $p \in P$ is the direct sum of the subspace $\gamma_{p}$ and $G(x)_{p}$, where $G(x)_{p}$ is the tangent space at the point $p$ of the fibre $G(x)$ over the point $x \in \rho(p)$;

2) The distribution $\gamma$ is right invariant: $R_{g}(\gamma)=\gamma^{11}$, where $R_{g}$ is a right translation of $P$ by $g \in G$.

The subspace $G(x)_{p}$ of $P_{p}$ is called the vertical subspace and $\gamma_{p}$ the horizontal subspace. According to 1 ), if we take a tangent vector $X \in P_{p}$, then we have the decomposition

$$
X=v(X)+h(X), \quad v(X) \in G(x)_{p}, h(X) \in \gamma_{p} .
$$

The vector $v(X)$ (resp. $h(X))$ is called the vertical (resp. horizontal) component of $X$.

We shall define next so-called connection forms of connections. We denote by $\hat{G}$ the Lie algebra of $G$, and identify $\hat{G}$ with the tangent space $G_{e}$ at the identity $e$ of $G$. Using the right translation $R_{g}$, we have the mapping

$$
L_{p}: G \rightarrow G(x), \quad L_{p}(g)=p \cdot g, p \in P, x=\rho(p) \in M .
$$

The fundamental vector field $A$ on $P$ corresponding to an element $\hat{A} \in \hat{G}$ is the vertical vector field, such that the value $A_{p}$ of $A$ at a point $p \in P$ is given by $L_{p}(\hat{A})$. Now, the connection form $\pi$ of the above connection $\gamma$ is a $\hat{G}$-valued differentiable 1 -form, which satisfies the following two conditions:

$\left.1^{*}\right)$ If $A$ is a fundamental vector field on $P$ corresponding to an element $\hat{A} \in \hat{G}$, then $\pi(A)=\hat{A}$.

$\left.2^{*}\right)$ For any horizontal vector $X \in \gamma_{p}$, we have $\pi(X)=0$.

It is well-known that $R_{g}(A)$ is the fundamental vector field

1) Later on, for the differential $\delta \varphi$ of a differentiable mapping $\varphi$, we shall use the same letter $\varphi$ in case there is no danger of confusion. The letter $\varphi^{*}$ means the dual of the differential $\delta \varphi$. 
corresponding to $\operatorname{ad}\left(g^{-1}\right) \cdot \hat{A} \in \hat{G}$, from which it follows the further property of $\pi$ as follows:

$\left.3^{*}\right)$ The connection form $\pi$ is of $a d(G)$-type : $R_{g}^{*}(\pi)=a d\left(g^{-1}\right) \cdot \pi$.

Conversely, given a differentiable 1 -form $\pi$ on $P$ with value in $\hat{G}$ satisfying $1^{*}$ ) and $3^{*}$ ), we can define a connection $\gamma$ whose connection form is this $\pi$. In this case, the horizontal subspace $\gamma_{p}$ at $p \in P$ is defined as the set of tangent vectors at $p$ which are mapped by $\pi$ into zero. This correspondence of connections and connection forms is one-to-one.

We shall treate mainly a connection $\mathrm{N}$ in the principal bundle $\Omega$, which is defined in the similar manner to the case of $\gamma$.

Theorem 1.1. Let $\eta$ be the canonical mapping $Q \rightarrow P$, and $\pi$ a connection form on $P$. Then the induced 1-form $\omega=\eta^{*}(\pi)$ is a connection form on $Q$.

Proof. It is sufficient to show that the similar conditions to $\left.1^{*}\right)$ and $3^{*}$ ) are also satisfied by $\omega$. Let $A^{\prime}$ be the fundamental vector field on $Q$ corresponding to $\hat{A} \in \hat{G}$. The value $A_{q}^{\prime}$ of $A^{\prime}$ at $q \in Q$ is $A_{q}^{\prime}=L_{q}^{\prime}(\hat{A})$, where the mapping $L_{q}^{\prime}$ is defined by means of $R_{g}^{\prime}$ in the similar manner to the case of $L_{p}$. In virtue of (1), we get $\eta \circ L_{q}^{\prime}=L_{p}, p=\eta(q)$, and hence we obtain

$$
\omega\left(A_{q}^{\prime}\right)=\pi\left(\eta \circ L_{q}^{\prime}(\hat{A})\right)=\pi\left(A_{p}\right)=\hat{A}, \quad p=\eta(q) .
$$

Next, using $\eta \circ R_{g}^{\prime}=R_{g} \circ \eta$, we get

$$
R_{g}^{\prime}(\omega)=\left(\eta \circ R_{g}^{\prime}\right) *(\pi)=\eta^{*} \circ R_{g}^{*}(\pi)=a d\left(g^{-1}\right) \cdot \omega .
$$

Thus it concludes the proof.

If there is a connection $\mathrm{I}^{\prime}$ in $\mathfrak{Q}$ and its connection form $\omega$ is the induced one by $\eta$ from the connection form $\pi$ of the connection $\gamma$ in $\mathfrak{B}$, then the connection $I^{\prime}$ and its connection form $\omega$ are called to be trivial.

We consider next a connection L' in $\Omega$, which is not necessarily to be trivial. The horizontal subspace $1_{q}$ of the tangent space $Q_{q}$ at $q \in Q$ is isomorphic to the tangent space $B_{b}$ at $b=\sigma(q)$ of the base $B$. By this isomorphism, for a tangent vector $X \in B_{b}$, we obtain the horizontal vector $l_{q}(X)$ at a point $q \in \sigma^{-1}(b)$, such that $X$ and $l_{q}(X)$ are $\sigma$ related : $\sigma\left(l_{q}(X)\right)=X$. The vector $l_{q}(X)$ is called the lift at $q$ of $X[7$, p. 26]. Let $X$ be a vertical vector at $b \in B$, that is, $\tau(X)=0$, and $B_{b}^{v}$ the set of vertical vectors at $b$. The set of lifts at $q \in \sigma^{-1}(b)$ of vertical vectors is clearly a subspace 
of the horizontal subspace $\Gamma_{q}$, which is called the zero-horizontal subspace of $Q_{q}$ and denoted by $\Gamma_{q}^{0}$. It is easily seen that $\tau \circ \sigma(\bar{X})=0$ for $\bar{X} \in \Gamma_{q}^{0}$.

Lemma 1.1. Let $\eta$ be the canonical mapping $Q \rightarrow P$.

1) The vertical subspace at $q \in Q$ is isomorphic to the vertical subspace at $\eta(q)=p \in P$ by the differential of $\eta$.

2) We suppose that a connection $\mathrm{L}^{\prime}$ is given in $\cong$. Then, for any zero-horizontal vector $\bar{X} \in \Gamma_{q}^{\prime o}$ at $q \in Q, \eta(\bar{X})$ is vertical at $\eta(q)=p \in P$.

Proof. 1) is a direct result from the definition of $\mathfrak{Q}$. From $\tau \circ \sigma=\rho \circ \eta$ it follows that $\tau \circ \sigma(\bar{X})=\rho \circ \eta(\bar{X})=0, \bar{X} \in \Gamma_{q}^{0}$. Hence $\eta(\bar{X})$ is vertical and this proves 2 ).

The trivial connections in $\mathfrak{Q}$ have particular properties among general connections in $\Omega$. We shall give some of them in the following theorem.

Theorem 1.2. Let $\mathrm{I}^{\prime}$ be a trivial connection in $\mathfrak{Q}$ induced from a connection $\gamma$ in $\mathfrak{B}$, and $\omega$ and $\pi$ the connection forms of $\Gamma$ and $\gamma$ respectively.

1) $\eta\left(\Gamma_{q}\right)=\gamma_{p}, p=\eta(q) \in P$ :

2) The connection form $\omega$ is central invariant: $C_{z}^{\prime *}(\omega)=\omega$, where $C_{z}^{\prime}$ is any central transformation of $Q$.

3) The kernel of the differential o $\eta$ is the zero-horizontal subspace $\Gamma^{0}$.

4) If we take $q=(b, p) \in Q$ and $v=p^{-1}(b) \in V$, and define $a$ mapping

$$
\mu_{v}: P \rightarrow Q, \quad \mu_{v}\left(p^{\prime}\right)=\left(p^{\prime}(v), p^{\prime}\right), \quad p^{\prime} \in P,
$$

then the horizontal subspace $\Gamma_{q}$ is the direct sum of $\mu_{v}\left(\gamma_{p}\right)$ and $\Gamma_{q}^{0}$.

Proof. 1) If we take $\bar{X} \in \Gamma_{q}$, then we have $\pi(\eta(\bar{X}))=\omega(\bar{X})=0$, and hence $\eta(\bar{X})$ is horizontal and thus we see $\eta\left(\Gamma_{q}\right) \subset \gamma_{p}$. Conversely, if we take $X \in \gamma_{p}$, then there exists $\bar{X} \in Q_{q}$ such that $\eta(\bar{X})=X$. Then we have $\eta(\bar{X})=\eta(v(\bar{X}))+\eta(h(\bar{X}))=X$. In virtue of Lemma $1.1,1)$, we see that $\eta(v(\bar{X}))$ is vertical. On the other hand, $\eta(h(\bar{X}))$ is horizontal as above shown. Since $X$ is horizontal, we have $\eta(v(\bar{X}))=0$, and hence $v(\bar{X})=0$. Therefore $\bar{X}$ is horizontal and consequently, we have $\eta\left(\Gamma_{q}\right)>\gamma_{p}$.

2) By means of $\eta \circ C_{z}^{\prime}=\eta$, we obtain

$$
C_{z}^{\prime *}(\omega)=\left(\eta \circ C_{z}^{\prime}\right) *(\pi)=\eta^{*}(\pi)=\omega .
$$

This prove 2). 
3) We take $\bar{X} \in Q_{q}$, such that $\eta(\bar{X})=0$. It is easily seen that $\sigma(\bar{X})$ is vertical at $b=\sigma(q) \in B$. If we take the lift $l_{q}(\sigma(\bar{X}))$ at $q$, then $\bar{X}-l_{q}(\sigma(\bar{X}))$ is vertical at $q$. On the other hand, $\eta(\bar{X})=$ $\eta(v(\bar{X}))+\eta(h(\bar{X}))=0$, and hence $\eta(v(\bar{X}))=0$, from which it follows that $v(\bar{X})=0$ and $\bar{X}$ is horizontal. Therefore $\bar{X}-l_{q}(\sigma(\bar{X}))$ is horizontal at $q$. Then we have $\bar{X}=l_{q}(\sigma(\bar{X}))$. Consequently the kernel of $\eta$ at $q \in Q$ is contained in $\Gamma_{q}^{0}$. Conversely, $\eta(\bar{X}), \bar{X} \in \Gamma_{q}^{0}$, is vertical by means of Lemma $1.1,2$ ), and horizontal by 1 ). Thus we have $\eta(\bar{X})=0$.

4) We shall first show that $\mu_{v}\left(\gamma_{p}\right) \subset \Gamma_{q}$. In fact, we take $X^{*} \in \gamma_{p}$ and then, according to $\eta^{\circ} \mu_{\nu}=$ identity, we have

$$
\omega\left(\mu_{v}\left(X^{*}\right)\right)=\pi\left(\eta \circ \mu_{v}\left(X^{*}\right)\right)=\pi\left(X^{*}\right)=0 .
$$

Hence $\mu_{v}\left(X^{*}\right)$ is horizontal at $q$. Next, let $\bar{X}$ be any horizontal vector at $q$. In virtue of 1$), \eta(\bar{X})$ is horizontal at $p=\eta(q)$ and hence $\mu_{v}^{\circ} \eta(\bar{X})$ is horizontal at $q$. Since $\eta\left(\bar{X}-\mu_{v} \circ \eta(\bar{X})\right)=0$, we have $\bar{X}-\mu_{v} \circ \eta(\bar{X})=\bar{Y} \in \Gamma_{a}^{0}$ by means of 3). Thus we obtain the decomposition $\mathrm{I}_{q}=\mu_{v}\left(\gamma_{p}\right)+\mathrm{I}_{q}^{0}$. With the aid of consideration of dimensions, we see easily that this decomposition is direct sum, and then we prove 4).

Theorem 1.3. The necessary and sufficient condition that $a$ connection $\Gamma$ in the principal bundle $\mathfrak{Q}$ be trivial is that

1) for any $q, q^{\prime} \in Q$, such that $\eta(q)=\eta\left(q^{\prime}\right)$, we obtain $\eta\left(\Gamma_{q}\right)=\eta\left(\Gamma_{q^{\prime}}\right)$.

2) $\eta\left(\Gamma_{q}^{0}\right)=0, q \in Q$.

Proof. The necessarity is clear from 1) and 2) of the above theorem, and hence we show the sufficiency. Given a connection $\Gamma$ in $\Omega$ satisfying the above conditions, we take a point $q \in Q$ for a given point $p \in P$, such that $\eta(q)=p$, and define $\gamma_{p}=\eta\left(\mathrm{L}_{q}^{\prime}\right)$. The subspace $\gamma_{p}$ of the tangent space $P_{p}$ is independent of the choice of a point $q \in Q, \eta(q)=p$, in consequence of 1$)$. We shall show that the distribution $\gamma: p \rightarrow \gamma_{p}$ of $P$ is a connection in $\mathfrak{\beta}$. For a tangent vector $X \in P_{p}$, we take a vector $\bar{X} \in Q_{q}$, such that $\eta(\bar{X})=X$. The vector $\bar{X}$ is written in the direct sum $\bar{X}=v(\bar{X})+h(\bar{X})$ with respect to the connection $\Gamma$. Then we obtain $X=\eta(v(\bar{X}))+\eta(h(\bar{X}))$, and $\eta(v(\bar{X}))$ is vertical in virtue of Lemma 1.1,1), while we see $\eta(h(\bar{X})) \in \gamma_{p}$ in consequence of the definition of $\gamma$. Thus we get the decomposition $P_{p}=G(x)_{p}+\gamma_{p}$. If we put $\operatorname{dim} . M=n$ and $\operatorname{dim} . V=v$, then we have dim. $\Gamma_{q}=n+v$ and dim. $\Gamma_{q}^{0}=v$. By applying 2) we see dim. $\gamma_{p} \leqq n$. Because of the possibility of the above decomposition, 
it follows that the decomposition is direct sum. Furthermore we obtain

$$
R_{g}\left(\gamma_{p}\right)=R_{g} \circ \eta\left(\Gamma_{q}\right)=\eta \circ R_{g}^{\prime}\left(\Gamma_{q}\right)=\eta\left(\mathrm{I}_{q \cdot g}^{\prime}\right)=\gamma_{p \cdot g},
$$

and hence $\gamma$ is right invariant. Therefore $\gamma$ is a connection in $\$$. It is easily seen that the original connection form $\omega$ on $Q$ is induced from the connection form $\pi$ of the connection $\gamma$ as above defined.

\section{Chapter II. Finsler connections}

\section{$\S 4$. Central invariant connections in the principal bundle $\mathfrak{Q}$}

In $\S 2$, we have defined the Finsler bundle $\Re *$ of the manifold $M$ constructed from the fibre bundle $\mathfrak{B}$ over $M$, and we have shown that $\bar{r}: \mathfrak{Q} \rightarrow \mathfrak{Q}^{*}$ is the bundle mapping. The Finsler connection of $M$ is defined to be a connection in the Finsler bundle $\mathfrak{\Omega}$.

On the other hand, a connection $\mathrm{I}$ in $\Omega$ is called to be central invariant, if $C_{z}^{\prime}\left(\mathrm{I}^{\prime}\right)=\Gamma$ for any central transformation $C_{z}^{\prime}$ of $Q$ by an element $z \in Z$. If we use its connection form $\omega$, then the central invariance of the connection is clearly expressed by $C_{z}^{\prime *}(\omega)=\omega$.

Theorem 2.1. There is a natural one-to-one correspondence between the set of central invariant connections in $\mathfrak{Q}$ and the set of Finsler connections in $\mathfrak{Q}^{*}$ by the bundle mapping $\bar{r}: \mathfrak{\Omega} \rightarrow \mathfrak{Q}$.

Before proving the theorem, we shall define the central-horizontal subspace $\Gamma_{q}^{z}, q \in Q$, of the zero-horizontal subspace $\Gamma_{q}^{0}$. We consider a point $b \in B$ and the equivalence class $r(b)=b^{*} \in B^{*}$. The point $b^{*}$ may be thought of as a submanifold of the fibre $V(x)$, $x=\tau(b)$, through the point $b$, and hence the set of the lifts at $q \in \sigma^{-1}(b)$ of tangent vectors at $b$ of the submanifold $b^{*}$ is a subspace $\Gamma_{q}^{z}$ of $\Gamma_{q}^{0}$, which is called the central-horizontal subspace at $q \in Q$. The mapping $r: B \rightarrow B^{*}$ is constant on $b^{*}$, and hence, in consequence of the definition of $\bar{r}$, we have immediately

Lemma 2.1. The differential of the bundle mapping $\bar{r}: Q \rightarrow Q^{*}$ carries a central-horizontal subspace to zero.

We shall prove now the theorem. Given a central invariant connection $\Gamma$ in $\mathscr{Q}$ and take a point $q \in Q$ for a given point $q^{*} \in Q^{*}$, such that $\bar{r}(q)=q^{*}$. We put $\mathrm{I}_{q}^{\prime} *=\bar{r}\left(\mathrm{I}_{q}\right)$. The subspace $\mathrm{I}_{q^{*}}$ is independent of the choice of a point $q \in Q, \bar{r}(q)=q^{*}$, in virtue of the central invariance of $\Gamma$. We shall show that the distribution $\Gamma^{*}: q^{*} \rightarrow \Gamma_{q^{*}}$ of $Q^{*}$ is a connection. In fact, given a tangent vector 
$X^{*}$ at $q^{*}$ of $Q^{*}$ and take $X \in Q_{q}$, such that $\bar{r}(X)=X^{*}$. Let $X=$ $v(X)+h(X)$ be the decomposition of $X$ with respect to $\Gamma$. Then we get $X^{*}=\bar{r}(v(X))+\bar{r}(h(X))$. The vector $\bar{r}(v(X))$ is clearly vertical, while $\bar{r}(h(X)) \in \mathrm{I}_{q^{*}}$ by the definition of $\mathrm{I}_{q} *$. That the above decomposition of $X^{*}$ is direct sum is immediately seen in consequence of Lemma 2.1. Next, let $R_{g}^{\prime \prime}$ be the right translation of $Q^{*}$ by $g \in G$, and then it follows that $R_{g}^{\prime \prime} \circ \bar{r}=\bar{r} \circ R_{g}^{\prime}$, where $R_{g}^{\prime}$ is the right translation of $Q$. Hence we obtain

$$
R_{g}^{\prime \prime}\left(\mathrm{I}^{\prime} *\right)=R_{g}^{\prime \prime} \circ \bar{r}\left(\mathrm{l}^{\prime}\right)=\bar{r} \circ R_{g}^{\prime}\left(\mathrm{I}^{\prime}\right)=\bar{r}\left(\mathrm{I}^{\prime}\right)=\mathrm{I}^{\prime} * .
$$

Therefore we prove that $\Gamma *$ is a Finsler connection.

Conversely, if we have a Finsler connection $\Gamma^{*}$, then we define $\omega=\bar{r}^{*}\left(\omega^{*}\right)$, where $\omega^{*}$ is the connection form of $\Gamma^{*}$. We shall show that $\omega$ is a connection form on $Q$. Let $A$ (resp. $A^{*}$ ) be the fundamental vector field on $Q$ (resp. $Q^{*}$ ) corresponding to $\hat{A} \in \hat{G}$. We see easily $\bar{r}(A)=A^{*}$, and then we obtain

$$
\omega(A)=\omega^{*}(\bar{r}(A))=\omega^{*}\left(A^{*}\right)=\hat{A} .
$$

Also we get, for a right translation $R_{g}^{\prime}$

$$
R_{g}^{\prime *}(\omega)=\left(\bar{r} \circ R_{g}^{\prime}\right)^{*}\left(\omega^{*}\right)=\bar{r}^{*} \circ R_{g}^{\prime \prime *}\left(\omega^{*}\right)=a d\left(g^{-1}\right) \cdot \omega .
$$

Hence the form $\omega$ is a connection form on $Q$. Next, for any central transformation $C_{z}^{\prime}$ of $Q$, we have

$$
C_{z}^{\prime *}(\omega)=\left(\bar{r} \circ C_{z}^{\prime}\right) *\left(\omega^{*}\right)=\bar{r}^{*}\left(\omega^{*}\right)=\omega,
$$

and thus $\omega$ is central invariant. Hence we have a central invariant connection L', whose connection form is $\omega$ as above defined. It is easily verified that the connection in $\mathfrak{2}$ constructed from $\Gamma$ by the process as shown in the first step coincides with the original Finsler connection $\Gamma *$. This concludes the proof of our theorem.

The structure of the principal bundle $\cong$ seems to be simpler than that of the Finsler bundle $\mathfrak{Q} *$, especially on local coordinates. And the above theorem shows that the study of the Finsler connection is equivalent to that of the central invariant connection of $\Omega$. Because of these points of view, our attention will be confined, in subsequent sections, to the principal bundle $\mathfrak{Q}$ with a central invariant connection. It is understood hereafter that $\Omega$ will be called the Finsler bundle of the manifold $M$ constructed from $\mathfrak{B}$ and a central invariant connection in $\cong$ a Finsler connection. From this standpoint, for a point $\tilde{b}=\left(b, b^{\prime}\right) \in B$, we shall say that the point $\tilde{\sigma}(\tilde{b})=b \in B$ is the element of support of $b^{\prime}$. 
As a result of Theorem 1.2, we see that a trivial connection of $\mathfrak{Q}$ is considered as one of the Finsler connection, and hence the existence of a Finsler connection is assured [7, p. 41]. By means of the well-known theorem [6, p. 68], we have

Theorem 2.2. Suppose that we have a trivial connection form $\omega_{0}$ in the Finsler bundle $\mathfrak{\Omega}$. Then there is an one-to-one correspondence between the set $\{\Gamma\}$ of Finsler connections and the set $\{\mu\}$ of $\hat{G}$-valued, ad $(G)$-type, central invariant, tensorial 1-forms on the bundle space $Q$, and the correspondence is given by

$$
\omega=\omega_{0}+\mu,
$$

where $\omega$ is the connection form of the connection $\Gamma \in\left\{I^{\prime}\right\}$ corresponding to the form $\mu \in\{\mu\}$.

We consider the local expressions of the connection form $\omega$. Let $U_{\infty}$ and $V_{\infty}=\tau^{-1}\left(U_{\infty}\right)$ be coordinate neighborhoods of $M$ and $B$ respectively, which were considered in $\S 1$. We suppose that we have a local section $\rho_{a}: U_{a} \rightarrow \rho^{-1}\left(U_{a}\right)$ of $\mathfrak{B}$, and then we obtain the induced local section $\sigma_{\alpha}: V_{a} \rightarrow \sigma^{-1}\left(V_{a}\right)$ of $\mathfrak{\Omega}$, such that

$$
\sigma_{a}(b)=\left(b, \quad \rho_{\infty} \circ \tau(b)\right), \quad b \in V_{a} .
$$

We denote by $\omega_{a}, \omega_{0 \omega}$ and $\mu_{a}$ the local expressions on $V_{\infty}$ of $\omega, \omega_{0}$ and $\mu$ respectively in the above theorem [6, pp. 60,67], which are defined by

$$
\omega_{\infty}=\sigma_{a}^{*}(\omega), \quad \omega_{0 \omega}=\sigma_{\alpha}^{*}\left(\omega_{0}\right), \quad \mu_{\infty}=\sigma_{a b}^{*}(\mu) .
$$

Let $\pi$ be the connection form on $P$, from which $\omega_{0}$ is induced, and $\pi_{a}$ the local expressions $\rho_{a}^{*}(\pi)$. Making use of $\eta \circ \sigma_{\infty}=\rho_{a} \circ \tau$, we get $\omega_{0 \alpha}=\tau^{*}\left(\pi_{a}\right)$. Thus we have the equations

$$
\omega_{a s}=\tau^{*}\left(\pi_{a}\right)+\mu_{a s} .
$$

It is easily verified that the forms $\omega_{a}, \pi^{*}\left(\pi_{\alpha}\right)$ and $\mu_{\infty}$ on $V_{\alpha}$ are all central invariant with respect to $C_{z}$.

\section{$\S 5$. Various parallel displacements}

Throughout the remainder of this chapter it is understood that we have a Finsler connection $\mathrm{I}^{\prime}$ in the Finsler bundle $\mathfrak{\Omega}$. Given a (piece-wise differentiable) curve $C=\left\{b_{t}, 0 \leqq t \leqq 1\right\}$ in the base space $B$, we have $a$ lift of $C$ to the bundle space $Q$ issuing from a point $q_{0}=\left(b_{0}, p_{0}\right) \in \sigma^{-1}\left(b_{0}\right)$, which is a horizontal curve $\bar{C}=\left\{q_{t}, 0 \leqq\right.$ $t \leqq 1\}$, such that $\sigma\left\{q_{t}\right)=b_{t}[7$, p. 27]. The existence and uniqueness 
are proved, and it is well-known that the curve $\bar{C} \cdot g, g \in G$, is also a lift of $C$ to $Q$ issuing from the point $q_{0} \cdot g$. We say that the end point $q_{1}$ of $\bar{C}$ is obtained from $q_{0}$ by parallel displacement along the curve $C$. Furthermore we have the definition of a holonomy group $\Phi_{q}$ with reference point at $q$, which is the set $\{g\}$ of elements of the structural group $G$, such that the point $q \cdot g, g \in\{g\}$, is obtained from $q$ by parallel displacement along a closed curve at $b=\sigma(q)$. Since the base space $B$ of $\mathfrak{Q}$ is the bundle space of the original fibre bundle $\mathfrak{B}$, we have a special subgroup $\Phi_{q}^{v}$ of the holonomy group $\Phi_{q}$. That is, if a curve $C$ in $B$ is contained in a fibre $V(x)$ over $x \in M$, then $C$ is said to be vertical. Then, the subgroup $\Phi_{q}^{v}$ is defined as the set of elements of $\Phi_{q}$ corresponding to all of closed vertical curve at $b=\sigma(q)$, and we shall call $\Phi_{q}^{v}$ the vertical subgroup of the holonomy group $\Phi_{q}$.

We shall define next the parallel displacement of points of $P$ along a curve $C=\left\{b_{t}, 0 \leqq t \leqq 1\right\}$ in $B$, where $P$ is the bundle space of $\mathfrak{F}$. Take a point $p_{0} \in \rho^{-1} \cdot \tau\left(b_{0}\right)$ of $P$, we have a point $q_{0}=\left(b_{0}\right.$, $\left.p_{0}\right) \in Q$. Then we obtain the lift $\bar{C}=\left\{q_{t}=\left(b_{t}, p_{t}\right), 0 \leqq t \leqq 1\right\}$ of $C$ to $Q$ issuing from $q_{0}$. Then we obtain the curve $\eta(\bar{C})=C^{*}=\left\{p_{t}, 0 \leqq t\right.$ $\leqq 1\}$ issuing from the given point $p_{0}$. The curve $C^{*}$ is determined by $C$ and the starting point $p_{0}$, and we call $C^{*}$ the lift of $C$ to $P$ issuing from $p_{0}$. It is clear that the lift of $C$ to $P$ issuing from $p=p_{0} \cdot g, g \in G$, is then given by $C^{*} \cdot g$. Now we say that the end point $p_{1}$ of the lift $C^{*}$ is obtained from $p_{0}$ by parallel displacement along the curve $C$.

We consider two curves $C=\left\{b_{t}, 0 \leqq t \leqq 1\right\}$ and $C^{\prime}=\left\{b_{t}^{\prime}, 0 \leqq t \leqq 1\right\}$ in $B$, such that there exists an element $z \in Z$ and $b_{t}^{\prime}=z \cdot b_{t}$ for any $t \in[0,1]$. Then $C$ and $C^{\prime}$ are called to be $z$-related. Since $\tau\left(b_{t}\right)$ $=\tau\left(b_{t}^{\prime}\right)=x_{t} \in M$, we can define the parallel displacements of a point $p_{0} \in \rho^{-1}\left(x_{0}\right)$ along both of $C$ and $C^{\prime}$. We take the lifts $\bar{C}=\left\{\left(b_{t}, p_{t}\right)\right\}$ and $\bar{C}^{\prime}=\left\{\left(b_{t}^{\prime}, p_{t}^{\prime}\right)\right\}$ to $Q$ of $C$ and $C^{\prime}$ respectively. Since the Finsler connection is central invariant, the curve $z \cdot \bar{C}=\left\{\left(b_{t}^{\prime}, p_{t}\right)\right\}$ is also horizontal and covers $C^{\prime}$. From the uniqueness of a lift, it follows that $z \cdot \bar{C}$ is to coincide with $\bar{C}^{\prime}$, and hence we obtain $p_{t}=p_{t}^{\prime}$. Therefore we have

Theorem 2.3. The parallel displacements of a point of $P$ along curves $C$ and $C^{\prime}$ coincide, if $C$ and $C^{\prime}$ are $z$-related.

We consider next the associated fibre bundle $\tilde{\mathfrak{B}}$ with the Finsler bundle $\mathfrak{Q}$ as defined in $\S 1$. The bundle space $\widetilde{B}$ of $\tilde{\mathfrak{B}}$ has 
been identified with $\bigcup_{x \in M} V(x) \times V(x)$. It is well-known [7, p. 43] that there is a natural one-to-one correspondence between the set of connections in $\mathfrak{Q}$ and the set of connections in $\tilde{\mathfrak{B}}$. Hence we have $a$ lift of a curve $C=\left\{b_{t}, 0 \leqq t \leqq 1\right\}$ in $B$ to $\widetilde{B}$, and we can define a parallel displacement of a point of $\widetilde{B}$ along $C$.

Let $\bar{C}=\left\{q_{t}=\left(b_{t}, p_{t}\right), 0 \leqq t \leqq 1\right\}$ and $\tilde{C}=\left\{\tilde{b}_{t}=\left(b_{t}, b_{t}^{\prime}\right), 0 \leqq t \leqq 1\right\}$ be lifts of $C$ to $Q$ and $\widetilde{B}$ respectively. The relation between $\bar{C}$ and $\tilde{C}$ is given by

$$
q_{t}^{\circ} q_{0}^{-1}\left(\tilde{b}_{0}\right)=\tilde{b}_{t}, \quad 0 \leqq t \leqq 1 .
$$

The end point $\tilde{b}_{1}$ of $\tilde{C}$ is said to be obtained from $\tilde{b}_{0}$ by parallel displacement along $C$. If we put $v_{0}=q_{0}^{-1}\left(\tilde{b}_{0}\right) \in V$, then we have $\left(b_{0}, b_{0}^{\prime}\right)=q_{0} v_{0}=\left(b_{0}, p_{0}\left(v_{0}\right)\right)$, and hence $v_{0}=p_{0}^{-1}\left(b_{0}^{\prime}\right)$. Thus we obtain $q_{t}^{\circ} q_{0}^{-1}\left(\tilde{b}_{0}\right)=q_{t} v_{0}=\left(b_{t}, p_{t}\left(v_{0}\right)\right)$. It follows from (4) that

$$
p_{t} \circ p_{0}^{-1}\left(b_{0}^{\prime}\right)=b_{t}^{\prime}, \quad 0 \leqq t \leqq 1,
$$

where $C^{*}=\left\{p_{t}, 0 \leqq t \leqq 1\right\}$ is the lift of $C$ to $P$.

In virtue of the equation (5), we can define the parallel displacement of points of $B$. Let $C$ be a curve in $B$ as above considered, and $b_{0}^{\prime}$ a point of the fibre through $b_{0}$, which is the starting point of $C$. Then we have a curve $C^{\prime}=\left\{b_{t}^{\prime}, 0 \leqq t \leqq 1\right\}$ in $B$ issuing from $b_{0}^{\prime}$, which is given by (5). We say that $C^{\prime}$ is parallel to $C$, and the end point $b_{1}^{\prime}$ of $C^{\prime}$ is obtained from $b_{0}^{\prime}$ by parallel displacement along the curve $C$ of its element of support $b_{0}$.

Theorem 2.4. If a curve $C^{\prime}$ in $B$ be parallel to a curve $C$ in $B$, then $C^{\prime}$ is also parallel to any $z$-related curve with $C$. That is, let $b_{1}^{\prime} \in B$ be obtained from $b_{0}^{\prime} \in B$ by the parallel displacement along $C$ of its element of support $b_{0}$. Then $b_{1}^{\prime}$ is obtained from $b_{0}^{\prime}$ by the parallel displacement along $z \cdot C, z \in Z$, of its element of support $z \cdot b_{0}$.

This theorem is a direct result from Theorem 2.3 and the equation (5).

Theorem 2.5. If a curve $C^{\prime}$ in $B$ be parallel to a curve $C$ in $B$, then a z-related curve with $C^{\prime}$ is also parallel to $C$. That is, let $b_{1}^{\prime} \in B$ be obtained from $b_{0}^{\prime} \in B$ by the parallel displacement along $C$ of its element of support $b_{0}$. Then a point $z \cdot b_{1}^{\prime}, z \in Z$, is obtained from $z \cdot b_{0}^{\prime}$ by the same parallel displacement along $C$.

Proof. From the definition of the central transformation $C_{z}$ of $B$, it follows that 


$$
\begin{array}{cl}
z \cdot p^{-1}(b)=p^{-1}(z, b), & p(z \cdot v)=z \cdot p(v), \quad \\
& p \in P, b \in B, v \in V, \\
& \rho(p)=\tau(b) .
\end{array}
$$

Making use of (5), we see that, for any $t$,

$$
p_{t} \circ p_{0}^{-1}\left(z \cdot b_{0}^{\prime}\right)=p_{t}\left(z \cdot p_{0}^{-1}\left(b_{0}^{\prime}\right)\right)=z \cdot\left(p_{t} \circ p_{0}^{-1}\left(b_{0}^{\prime}\right)\right)=z \cdot b_{t}^{\prime} .
$$

This proves the theorem.

\section{§6. Property $D$ of a Finsler connection}

Let $C=\left\{b_{t}, 0 \leqq t \leqq 1\right\}$ be a vertical curve contained in the fiber $V\left(x_{0}\right)$ in $B$, and $\bar{C}=\left\{q_{t}=\left(b_{t}, p_{t}\right), 0 \leqq t \leqq 1\right\}$ the lift of $C$ to $Q$. Since we have

$$
\rho\left(p_{t}\right)=\rho \circ \eta\left(q_{t}\right)=\tau \circ \sigma\left(q_{t}\right)=\tau\left(b_{t}\right)=x_{0},
$$

the lift $C^{*}=\eta(\bar{C})$ of $C$ to $P$ is also vertical and contained in the fibre $G\left(x_{0}\right)$. Therefore there exists a curve $C_{G}=\left\{g_{t}, 0 \leqq t \leqq 1\right\}$ in the structural group $G$, such that $p_{t}=p_{0} \cdot g_{t}$ for any $t$. The curve $C_{G}$ is called the development of the vertical curve $C$ with reference point at $p_{0} \in P$. It is clear that any development of $C$ in $G$ is given by $g^{-1} \cdot C_{G} \cdot g, g \in G$. On the other hand, if we take a curve $C^{\prime}$ in $B$, which is parallel to $C$, then $C^{\prime}$ is also vertical and contained in the above fibre $V\left(x_{0}\right)$.

Now, in order to determine so-called euclidean connection in a Finsler manifold by means of the fundamental function, E. Cartan introduced five postulates $A, B, C, D$ and $E[2, \mathrm{p} .10]$. The fourth of them is expressible as follows [11, p. 68]:

"If the direction of a vector with fixed components coincides with that of its element of support, then its covariant differential corresponding to an infinitesimal rotation of its element of support about its own centre vanishes identically".

In our case of general Finsler bundles, the centre of the element of support $b \in B$ of a point $b^{\prime} \in B$ is to be defined as the point $x=\tau(b) \ni M$, and hence the rotation of $b$ means that $b$ moves along a vertical curve in $B$. Therefore we shall give the property $D$ of a Finsler connection as follows:

Definition. The Finsler connection is said to have the property $D$, when any curve issuing from $a$ point $b_{0}$ and being parallel to $a$ vertical curve $C$ is a single point $b_{0}$, if the starting point of $C$ coincides with $b_{0}$.

That is, a point obtained from $b_{0}$ by parallel displacement 
along any vertical curve $C$ of the element of support $b_{0}$ coincides with the original point $b_{0}$ at any time $t$. Hence, by virtue of (5), the analytic expression of the property $D$ is that

$$
p_{t} \circ p_{0}^{-1}\left(b_{0}\right)=b_{0},
$$

where $C^{*}=\left\{p_{t}, 0 \leqq t \leqq 1\right\}$ is a lift to $P$ of a vertical curve $C=\left\{b_{t}\right.$, $0 \leqq t \leqq 1\}$ issuing from $b_{0}$. In this case $C^{*}$ is also vertical and expressed as $p_{t}=p_{0} \cdot g_{t}$, where $C_{G}=\left\{g_{t}, 0 \leqq t \leqq 1\right\}$ is the development of $C$ in $G$ with the reference point $p_{0} \in P$. If we put $p_{0}^{-1}\left(b_{0}\right)=v_{0} \in V$, then (6) is written in the form $p_{0}\left(g_{t} \cdot v_{0}\right)=p_{0}\left(v_{0}\right)$, and hence we get $g_{t} \cdot v_{0}=v_{0}$. Consequently the development $C_{G}$ is a curve in an isotropy subgroup $G\left(v_{0}\right)$ of $G$, the elements of which map $v_{0}$ into itself. It is clear that any development $g^{-1} \cdot C_{G} \cdot g$ is contained in an isotropy subgroup $G\left(g^{-1} \cdot v_{0}\right)$.

Conversely, if a development $C_{G}=\left\{g_{t}, 0 \leqq t \leqq 1\right\}$ of any vertical curve $C=\left\{b_{t}, 0 \leqq t \leqq 1\right\}$ with reference point $p_{0}$ be contained in an isotropy subgroup $G^{\prime}\left(v_{0}\right), v_{0}=p_{0}^{-1}\left(b_{0}\right)$, of $G$, then we take a lift $C^{*}=\left\{p_{t}, 0 \leqq t \leqq 1\right\}$ to $P$ issuing from $p_{0}$, and we have $p_{t}=p_{0} \cdot g_{t}$, and hence

$$
p_{t} \cdot p_{0}^{-1}\left(b_{0}\right)=p_{0}\left(g_{t} \cdot v_{0}\right)=p_{0}\left(v_{0}\right)=b_{0},
$$

from which it follows that (6) is satisfied. Thus we establish

Theorem 2.6. A Finsler connection has the property $D$, if and only if a development of any vertical curve in $B$ is a curve contained in an isotropy subgroup $G(v), v \in V$, of the structural group $G$.

In the last section we have defined the vertical subgroup $\Phi_{q}^{v}$ of the holonomy group $\Phi_{q}$. From the above theorem we have

Corollary. If a Finsler connection has the property $D$, then the vertical subgroup of the holonomy group with reference point $q=(b, p)$ is contained in an isotropy subgroup $G(v)$ of $G$, where $v=p^{-1}(b)$.

\section{Chapter III. Linear Finsler connections}

\section{$\S 7$. Canonical coordinates}

Throughout the remainder of this paper we shall confine ourself to the case where the original fibre bundle $\mathfrak{B}$ is the tangent bundle of the $n$-dimensional differentiable manifold $M$, so that the structural Lie group is the general linear group $G L(n, R)$ and the standard fibre $V$ is the $n$-dimensional vector space over the real field $R$. Then the associated principal bundle is the bundle of 
frames on $M$, and a point $q=(b, p)$ of the bundle space $Q$ of the Finsler bundle $\mathscr{Q}$ is a pair of a tangent vector $b \in B$ and a frame $p=\left(p_{1}, \cdots, p_{n}\right) \in P$, such that $b, p_{1}, \cdots, p_{n}$ are tangent vectors at the same point of $M$. Then $\mathfrak{Q}$ is called the linear Finsler bundle of $M$. A point of the bundle space $\widetilde{B}$ of the associated bundle $\tilde{B}$ with $\Omega$ can be considered as a pair of tangent vectors at a point of $M$. In the following it is understood that the element of support $b$ of $b^{\prime}$ is not a zero vector.

The centre of $G L(n, R)$ is the set of $n$-matrices, which are expressed in the forms $\left((z) \cdot \delta_{j}^{i}\right)$, where $(z) \in R-0$ and $\delta_{j}^{i},(i, j=1$, $\cdots, n)$ are the Kronecker's deltas. Thus there is an one-to-one correspondence between elements of $Z$ and real numbers $\neq 0$. We denote by $(z)$ the real number corresponding to an element $z \in Z$.

Let $\left(x^{i}\right),(i=1, \cdots, n)$ be the local coordinates of a point in the coordinate neighborhood $U_{a}$ of $M$, and $\left(e_{i}\right),(i=1, \cdots, n)$ a fixed base of $V$. A tangent vector $b \in B$ at $\tau(b)=x \in U_{a}$ is expressed by the canonical frame $\left(\partial / \partial x^{i}\right)$ in the form $b=b^{i}\left(\partial / \partial x^{i}\right)_{x}{ }^{2)}$ and hence $\left(x^{i}, b^{i}\right)$ are considered as coordinates of a point in $V_{\alpha}=\tau^{-1}\left(U_{a}\right)$ of the base space $B$ of $\mathfrak{Q}$. We shall call $\left(x^{i}, b^{i}\right)$ the canonical coordinates in $V_{\infty}$. On the other hand, a vector $p_{i},(i=1, \cdots, n)$, of a frame $p=\left(p_{1}, \cdots, p_{n}\right) \in p, \rho(p)=x \in V_{a}$, is expressible in the form $p_{i}=p_{i}^{j}\left(\partial / \partial x^{j}\right)_{x}$, and hence a point $q=(b, p) \in Q, b \in V_{a}$, is expressed by the set of real numbers $\left(x^{i}, b^{i}, p_{i}^{j}\right)$, which are called also the canonical coordinates in $\sigma^{-1}\left(V_{a}\right)$. If we take $q=(b, p)=\left(x^{i}, b^{i}, p_{i}^{j}\right) \in Q$, then $p^{-1}(b)=v \in V$ is of the form $v=p_{j}^{-1 t} b^{j} \cdot e_{i}$, where the matrix $\left(p_{j}^{-1 i}\right)$ is the inverse of $\left(p_{i}^{j}\right)$. Thus the central transformation $C_{z}$ of $B$ by $z \in Z$ is given by

$$
C_{z}(b)=\left(x^{i},(z) b^{i}\right), \quad \text { where } b=\left(x^{i}, b^{i}\right) .
$$

Now we consider a connection form $\pi$ on $P$. It is well known $\left[6\right.$, p. 85] that the local expressions $\pi_{a}$ of $\pi$ are given by

$$
\pi_{a}=\gamma_{j_{k}}^{i}\left(x^{1}, \cdots, x^{n}\right) d x^{k} \cdot \hat{g}_{i}^{j}, \quad i, j, k=1, \cdots, n,
$$

where $\left(\hat{g}_{i}^{j}\right)$ is a fixed base of the Lie algebra of $G L(n, R)$. If we write the 1 -form $\mu_{\infty}$ in the equations (3) by

$$
\begin{gathered}
\mu_{\infty}=\left[\mu_{j_{k}}^{i}\left(x^{1}, \cdots, x^{n}, b^{1}, \cdots, b^{n}\right) d x^{k}+C_{j_{k}}^{i}\left(x^{1}, \cdots, x^{n}, b^{1}, \cdots, b^{n}\right) d b^{k}\right] \cdot \hat{g}_{i}^{\jmath}, \\
i, j, k=1, \cdots, n,
\end{gathered}
$$

2) We make free use of the summation convention. 
then, in virtue of (3), the local expressions $\omega_{a s}$ of a general linear Finsler connection form $\omega$ are expressed in terms of canonical coordinates as follows:

$$
\omega_{\infty}=\left(\Gamma_{j_{k}}^{i} d x^{k}+C_{j_{k}}^{i} d b^{k}\right) \cdot \hat{g}_{i}^{j}, \quad i, j, k=1, \cdots, n,
$$

where we put

$$
\Gamma_{j_{k}}^{i}=\gamma_{j_{k}}^{i}\left(x^{1}, \cdots, x^{n}\right)+\mu_{j_{k}}^{i}\left(x^{1}, \cdots, x^{n}, b^{1}, \cdots, b^{n}\right) .
$$

Since $\mu_{a}$ is central invariant, it is immediately verified that, with respect to the variables $b^{1}, \cdots, b^{n}$, the coefficients $\mu_{j k}^{i}$ and $C_{j k}^{i}$ are homogeneous of degree 0 and -1 respectively. Therefore $\omega_{\infty}$ as above are the local expressions of Finsler connection form, if and only if, with respect to the variables $b^{1}, \cdots, b^{n}$, the coefficients $\Gamma_{j_{k}}^{i}$ and $C_{j k}^{i}$ are homogeneous of degree 0 and -1 respectively, and further the well-known formula of transformation [6, p. 60] is satisfied.

It can be easily shown that the lift $l_{q}(X)$ of a tangent vector $X=X^{i}\left(\partial / \partial x^{i}\right)_{b}+X^{(i)}\left(\partial / \partial b^{i}\right)_{b}$ with respect to the Finsler connection (7) is given by

$$
\begin{array}{r}
l_{q}(X)=X^{i}\left(\frac{\partial}{\partial x^{i}}\right)_{q}+X^{(i)}\left(\frac{\partial}{\partial b^{i}}\right)_{q}-p_{j}^{k}\left(\Gamma_{k l}^{i}(b) X^{l}\right. \\
\left.+C_{k l}^{i}(b) X^{(l)}\right)\left(\frac{\partial}{\partial p_{j}^{i}}\right)_{q}, \\
\quad i, j, k, l=1, \cdots, n .
\end{array}
$$

Now, we shall treat parallel displacements. Let $C: b_{t}=\left(x^{i}(t)\right.$, $\left.b^{i}(t)\right)$ be a curve in $B$. Making use of (8), we have the condition for $\bar{C}: q_{t}=\left(x^{i}(t), b^{i}(t), p_{j}^{i}(t)\right)$ to be a lift of $C$ as follows:

$$
\frac{d p_{j}^{i}}{d t}+p_{j}^{k}\left(\Gamma_{k l}^{i}(b) \frac{d x^{l}}{d t}+C_{k l}^{i}(b) \frac{d b^{l}}{d \bar{t}}\right)=0 .
$$

In particular, when $C$ is vertical, then $x^{i}(t), i=1, \cdots, n$, are identical to $x^{i}(o)$, and $p_{j}^{i}(t), i, j=1, \cdots, n$, are written in the forms $p_{j}^{i}(t)=$ $p_{k}^{i}(o) \cdot g_{j}^{k}(t)$, where $C_{G}: g_{t}=\left(g_{j}^{k}(t)\right)$ is a development of $C$ in $G L(n, R)$. Hence the lift $\bar{C}$ is given by

$$
p_{k}^{i}(o) \frac{d g_{j}^{k}}{d t}+p_{h}^{k}(o) g_{j}^{h} C_{k l}^{i}\left(b_{t}\right) \frac{d b^{l}}{d t}=0 .
$$

We consider the property $D$ of a Finsler connection. In consequence of Theorem 2.6, given a vertical curve $C: b_{t}=\left(x_{o}^{i}, b^{i}(t)\right)$, we take its development $C_{G}: g_{t}=\left(g_{j}^{i}(t)\right)$ with reference point $\left(x_{o}^{i}, p_{j o}^{i}\right)$ and 
a vector $v=v^{i} \cdot e_{i}$, where $v^{i}=p_{j_{0}}^{-1 i} b^{j}(o)$. Then we obtain $g_{j}^{k}(t) v^{j}=v^{k}$, and hence contraction of (10) by $v^{j}$ gives easily

$$
b^{j} C_{j_{k}}^{i}(b)=0, \quad i, j, k=1, \cdots, n,
$$

which is the well-known equation [4, (10)], [11, p. 69]. Thus we get

Theorem 3.1. In terms of canonical coordinates, the property $D$ of the Finsler connection (7) is written in the form (11).

Finally we shall find the geometrical meaning of the wellknown equation $[11$, p. 15$]$

$$
C_{j k}^{i}(b) b^{k}=0, \quad i, j, k=1, \cdots, n .
$$

For this purpose, we consider curves $C=\left\{b_{t}\right\}$ and $C^{\prime}=\left\{b_{t}^{\prime}\right\}$ in $B$. If there exists a curve $C_{Z}=\left\{z_{t}\right\}$ in $Z$, such that $b_{t}^{\prime}=z_{t} \cdot b_{t}$ for any $t$, then we say that $C$ and $C^{\prime}$ are central-related. In particular, when $C_{z}$ is a single point $z$, then $C$ and $C^{\prime}$ are simply $z$-related, as defined in $\S 5$. We shall show that

Theorem 3.2. Let $C^{\prime}$ be a curve in $B$, which is parallel to a curve $C$ in $B$. The curve $C^{\prime}$ is also parallel to any curve, which is cental-related with $C$, if and only if (12) be satisfied.

Proof. First we shall find the equation which shows that $C^{\prime}$ is parallel to $C$. If we put $C: b_{t}=\left(x^{i}(t), b^{i}(t)\right)$ and $C^{\prime}: b_{t}^{\prime}=\left(x^{i}(t)\right.$, $\left.b^{\prime i}(t)\right)$, then we have from (5) that

$$
p_{j}^{i}(t) p_{k}^{-1 j}(o) b^{\prime k}(o)=b^{\prime i}(t),
$$

where $p_{j}^{i}(t)$ satisfy (9). Therefore we have

$$
\frac{d b^{\prime i}}{d t}+b^{\prime k}\left(\Gamma_{k l}^{i}(b) \frac{d x^{l}}{d t}+C_{k l}^{i}(b) \frac{d b^{l}}{d t}\right)=0 .
$$

This is the condition that $C^{\prime}$ be parallel to $C[4,(11)]$. Now we take a central-related curve $\bar{C}: b_{t}=\left(x^{i}(t),(z(t)) b^{i}(t)\right)$ with $C$ and then, in virtue of (13), the condition that $C^{\prime}$ be also parallel to $\bar{C}$ is given by $b^{\prime k} C_{k l}^{l}(b) b^{l}\left((z)^{-1}\right)(d(z) / d t)=0$, and hence the theorem is immediately proved.

\section{§8. Vertical vector bundle and characteristic vector field}

We have denoted, in $\S 3$, by $B_{b}^{o}$ the set of vertical vectors at a point $b$ of $B$. We put now $B^{v}=\bigcup_{b \in B} B_{b}^{v}$ and then define a mapping $\lambda: \widetilde{B}=\bigcup_{x \in M} V(x) \times V(x) \rightarrow B^{v}$ as follows : 


$$
\lambda\left(\left(b, b^{\prime}\right)\right)=\delta p\left(\left(p^{-1}\left(b^{\prime}\right)\right)_{n}\right) \in B_{b}^{n}, \quad p \in \sigma^{-1} \circ \tau(b), \quad v=p^{-1}(b) .
$$

The letters in the right hand side will be explained in the following. Given a point $\tilde{b}=\left(b, b^{\prime}\right) \in \widetilde{B}$, we take an arbitrary point $p \in \rho^{-1} \circ \tau(b)$, which is considered as an admissible mapping of $V$ onto $V(x)$. Hence $p^{-1}\left(b^{\prime}\right)$ is a point of $V$, namely, a vector, so that we can identify $p^{-1}\left(b^{\prime}\right)$ with a tangent vector at $v=p^{-1}(b) \in V$ in the ordinary manner, and denote it by $\left(p^{-1}\left(b^{\prime}\right)\right)_{v}$. Then the differential $\delta p$ of the admissible mapping $p$ maps this to the vertical vector at $b$ of $B$. It is easily verified that the vertical vector as thus obtained is independent of the choice of $p$.

Conversely, given a vertical vector $X \in B_{b}^{v}$, we have a tangent vector $\delta p^{-1}(X)$ at $v=p^{-1}(b)$, and then can identify with a point of $V$. Hence $p\left(\delta p^{-1}(X)\right)$ is a point of the fibre through $b$, and thus we obtain the point $\left(b, p\left(\delta p^{-1}(X)\right)\right) \in \widetilde{B}$. It is easily seen that $\lambda((b$, $\left.\left.p\left(\delta p^{-1}(X)\right)\right)\right)=X$. Therefore $\tilde{B}$ can be identified with $B^{v}$ by the mapping $\lambda$, and we shall call $\tilde{\mathfrak{B}}$ the vertical tangent bundle of $B$.

Next, we consider a tangent vector $X \in B_{b}$ and put $X^{v}=\lambda((b$, $\tau(X))$ ), where $\tau(X)$ is a tangent vector at $x=\tau(b)$ of $M$, which is considered as a point of the fibre over $x$. Thus, for a vector $X \in B_{b}$, we have an unique vertical vector $X^{v}$, which will be called the induced vertical vector from $X$. In terms of canonical coordinates, if $X=X^{i}\left(\partial / \partial x^{i}\right)_{b}+X^{(i)}\left(\partial / \partial b^{i}\right)_{b}$, then we have $X^{v}=X^{i}\left(\partial / \partial b^{i}\right)_{b}$. The next lemma is a direct result from the definition $X \rightarrow X^{v}$.

Lemma 3.1. Let $X$ and $Y \in B_{b}$, and $f$ be a real function on $B$.

1) The mapping $X \rightarrow X^{v}$ is linear:

$$
(X+Y)^{v}=X^{v}+Y^{v}, \quad(f \cdot X)^{v}=f \cdot X^{v} .
$$

2) $X^{v}=0$, if and only if $X$ be vertical.

The relation between the mapping $X \rightarrow X^{v}$ and central transformations of $B$ is given by the following lemma.

Lemma 3.2. Let $C_{z}$ be a central transformation of $B$ by $z \in Z$. Then, for $X \in B_{b}$, we obtain $\left(C_{z}(X)\right)^{v}=(z)^{-1} \cdot C_{z}\left(X^{v}\right)$.

Proof. If we identify a point $u \in V$ with a tangent vector $u_{v}$ at $v \in V$, then it is immediately obtained that

$$
g \cdot u_{v}=(g \cdot u)_{g \cdot v} .
$$

It follows from (14) and the definition of $X \rightarrow X^{v}$, that, for $X \in B_{b}$, $p \in \rho^{-1} \circ \tau(b)$, and $v=p^{-1}(b)$, 


$$
\begin{aligned}
& \left(C_{z}(X)\right)^{v}=\delta p\left(\left(p^{-1} \circ \tau\left(C_{z}(X)\right)\right)_{z \cdot v}\right)=\delta p\left(\left(p^{-1} \circ \tau(X)\right)_{z \cdot v}\right) \\
& =\delta p\left(z \cdot\left(z^{-1} \cdot p^{-1}(\tau(X))\right)_{v}\right)=C_{z} \circ \delta p\left(\left(z^{-1} \cdot p^{-1}(\tau(X))\right)_{v}\right)=C_{z}\left((z)^{-1} \cdot X^{v}\right) .
\end{aligned}
$$

Thus we have proved the lemma.

We shall now define a special vertical vector field on $B$, which will play a röle in the following. Let $\tilde{b}=(b, b)$ be a diagonal point of $\widetilde{B}$, and then we have a vertical vector $\lambda((b, b))$ at $b$ of $B$, which will be denoted by $\vec{b}$. The vector field $b \in B \rightarrow \vec{b}$ is called the characteristic vector field on $B$. In terms of canonical coordinates, the vector $\vec{b}$ at $b$ is given by $b^{i}\left(\partial / \partial b^{i}\right)_{b}$, where $b=\left(x^{i}, b^{i}\right)$.

Lemma 3. 3. The characteristic vector field $\vec{b}$ is invariant by central transformations of $B$.

Proof. In virtue of the definition of $\vec{b}$ and (14), we get

$$
C_{z}(\vec{b})=C_{z} \circ \delta p\left(v_{v}\right)=\delta p\left(z \cdot v_{v}\right)=\delta p\left((z \cdot v)_{z^{*} v}\right)=(\overrightarrow{z \cdot b}),
$$

where $v=p^{-1}(b)$. This prove the lemma.

The following two lemmas can be easily verified, using canonical coordinates.

Lemma 3.4. Let $X$ and $Y$ be vector fields on $B$. Then the bracket of $X^{v}$ and $Y^{v}$ is written as follows:

$$
\left[X^{v}, Y^{v}\right]=\left[X, Y^{v}\right]^{v}+\left[X^{v}, Y\right]^{v} .
$$

Lemma 3.5. Let $X$ be a vector field on $B$ and $\vec{b}$ the characteristic vector field on $B$. Then we obtain

$$
[\vec{b}, X]^{v}=\left[\vec{b}, X^{v}\right]+X^{v} .
$$

The inverse mapping of $X \rightarrow X^{v}$ is not uniquely determined. But we shall denote by $X^{h}$ a vector, from which the vertical vector $X$ is induced. By means of Lemma 3.1,2), we see that such a vector $X^{h}$ is determined within vertical vector. $X^{h}$ is called $a$ vector inducing the vector $X$.

Lemma 3.6. Let $X$ be a vector field on $B$, and then the vector $X+\left[\vec{b}^{h}, X^{v}\right]$ is vertical.

Proof. In consequence of Lemma 3.1, it is sufficient to show that $X^{v}+\left[\overrightarrow{b^{h}}, X^{v}\right]^{v}=0$ identically. It follows from Lemma 3.4 that

$$
\left[\vec{b}, X^{v}\right]=\left[\overrightarrow{b^{h}}, X^{v}\right]^{v}+[\vec{b}, X]^{v} .
$$

Then the lemma is assured by Lemma 3.5. 


\section{§9. Horizontal forms and tensors}

Let $\alpha$ be a $s(\geqq 1)$-form $B$ and $X_{1}, \cdots, X_{s} \in B_{b}$, such that one at least of $X_{1}, \cdots, X_{s}$ is vertical. If $\alpha\left(X_{1}, \cdots, X_{s}\right)=0$ for any point $b$ of $B$, then $\alpha$ will be called to be horizontal. For such a form $\alpha$, we put

$$
\alpha_{0}\left(X_{2}, \cdots, X_{s}\right)=\alpha\left(\vec{b}^{h}, X_{2}, \cdots, X_{s}\right), \quad X_{2}, \cdots, X_{s} \in B_{b} .
$$

Since $\alpha$ is horizontal, the $(s-1)$-form $\alpha_{0}$ as thus defined is independent of the choice of $\vec{b}^{h}$. The form $\alpha_{0}$ will be called the reduced form from $\alpha$ for $s>1$, which is clearly horizontal. If $s=1$, then $\alpha_{0}$ will be called the reduced function from $\alpha$ [2, p. 18]. For $s>1$, we get

$$
\alpha\left(X_{1}, \cdots, \vec{b}_{(i)}^{h}, \cdots, X_{s}\right)=(-1)^{i-1} \cdot \alpha_{0}\left(X_{1}, \cdots, \hat{X}_{i}, \cdots, X_{s}\right)^{3)},
$$

where $X_{1}, \cdots, \hat{X}_{i}, \cdots, X_{s} \in B_{b}$.

Next, let $\underline{X}_{1}, \cdots, \underline{X}_{s} \in M_{x}$ be a set of tangent vectors at $x$. Then we have tangent vectors $X_{1}, \cdots, X_{s} \in B_{b}, b \in V(x)$, such that $\tau\left(X_{i}\right)=\underline{X}_{i}, i=1, \cdots, s$. Such $X_{i}$ are determined within vertical vectors, and hence we can define $\alpha(b)$ uniquely as follows:

$$
\alpha(b)\left(X_{1}, \cdots, \underline{X}_{s}\right)=\alpha\left(X_{1}, \cdots, X_{s}\right) .
$$

The mapping $\alpha(b)$ as thus defined will be called the projection of the horizontal form $\alpha$.

Next, we consider a $s(\geqq 1)$-form $\beta$ on $B$, which is not necessarily to be horizontal. If we put

$$
\beta^{h}\left(X_{1}, \cdots, X_{s}\right)=\beta\left(X_{1}^{v}, \cdots, X_{s}^{v}\right), \quad X_{1}, \cdots, X_{s} \in B_{b},
$$

then we obtain a $s$-form $\beta^{h}$ on $B$, which is horizontal by virtue of Lemma 3.1,2). We shall call $\beta^{h}$ the horizontal form induced from $\beta$. It is clear that

Lemma 3.7. If $\alpha$ is a horizontal $s(\geqq 1)$-form on $B$, then the induced horizontal form $\alpha^{h}$ vanishes identically.

The above notions and processes for forms on $B$ can be applied equally well to covariant $s(\geqq 1)$-tensors on $B$. We can define notions of a horizontal tensor and induced horizontal tensor, and further we obtain the projection of a tensor. On the other hand,

3) The sign ( $i)$ under a letter indicates that we replace $X_{i}$ to this letter, and the sign $(\wedge)$ over a letter does that this letter is to be omitted. 
in the case of horizontal covariant $s$-tensor $T$, we have to remark that $T$ is not necessarily skew-symmetric, so that we obtain from $T$ reduced tensors of different type, and hence we shall write

$$
\begin{gathered}
T_{0(i)}\left(X_{1}, \cdots, \hat{X}_{i}, \cdots, X_{s}\right)=T\left(X_{1}, \cdots, \vec{b}_{(i)}^{h}, \cdots, X_{s}\right), \\
X, \cdots, \hat{X}_{i}, \cdots, X_{s} \in B_{b} .
\end{gathered}
$$

\section{$\S 10$. Covariant vertical derivatives}

E. Cartan introduced a simple process of a covariant differentiation [2, p. 12]. We shall define such a process in our case. In the first place, we consider a real function $f$ on $B$, and put

$$
\Delta^{v} f(X)=X^{v}(f), \quad X \in B_{b} .
$$

This covariant vector $\Delta^{v} f$ is clearly horizontal in virtue of Lemma 3.1 , which will be called the covariant vertical derivative, or, for brevity, covariant $v$-derivative of $f$.

Generally, let $T$ be a horizontal covariant $s(\geqq 1)$-tensor on $B$ and put, for $X_{1}, \cdots, X_{s}, Y \in B_{b}$,

$$
\begin{aligned}
& \Delta^{v} T\left(X_{1}, \cdots, X_{s}, Y\right) \\
& \quad=Y^{v}\left(T\left(X_{1}, \cdots, X_{s}\right)\right)+\sum_{i=1}^{s} T\left(X_{1}, \cdots,\left[X_{i}, Y^{v}\right], \cdots, X_{s}\right)
\end{aligned}
$$

We shall verify that $\Delta^{v} T$ as thus defined is a convariant $(s+1)$ tensor. In fact, we can easily show that

$\Delta^{v} T\left(X+X^{\prime}, X_{2}, \cdots, X_{s}, Y\right)=\Delta^{v} T\left(X, \cdots, X_{s}, Y\right)+\Delta^{v} T\left(X^{\prime}, \cdots, X_{s}, Y\right)$, $\Delta^{v} T\left(X_{1}, \cdots, X_{s}, Y+Y^{\prime}\right)=\Delta^{v} T\left(X_{1}, \cdots, X_{s}, Y\right)+\Delta^{v} T\left(X_{1}, \cdots, X_{s}, Y^{\prime}\right)$,

where $X, X^{\prime}, X_{2}, \cdots, X_{s}, Y, Y^{\prime} \in B_{b}$. Next, let $\rho$ and $\psi$ be functions on $B$, and $X$ and $Y$ vector fields on $B$. Then it is well-known [7, p. 4] that

$$
[\varphi X, \psi Y]=\mathscr{P} \cdot \psi \cdot[X, Y]+\boldsymbol{\rho} \cdot(X(\psi)) \cdot Y-\psi \cdot(Y(\mathcal{P})) \cdot X .
$$

Making use of this we obtain immediately, for $X_{1}, \cdots, X_{s}, Y \in B_{b}$,

$$
\begin{aligned}
& \Delta^{v} T\left(\rho X_{1}, X_{2}, \cdots, X_{s}, \psi Y\right)=\varphi \cdot \psi \cdot \Delta^{v} T\left(X_{1}, \cdots, X_{s}, Y\right) \\
& \quad+\varphi \cdot\left(X_{1}(\psi)\right) \cdot T\left(Y^{v}, X_{2}, \cdots, X_{s}\right)+\varphi \cdot \sum_{i=2}^{s} X_{i}(\psi) \cdot T\left(X_{1}, \cdots, Y_{(i)}^{v}, \cdots, X_{s}\right) .
\end{aligned}
$$

Since $T$ is assumed to be horizontal, then the last two terms of the above equation vanish, and hence we conclude that $\Delta^{v} T$ is a covariant tensor. Since $\left[X_{i}, Y^{v}\right]$ is vertical for any vertical 
vector $X_{i}$, then it is easily seen that $\Delta^{v} T$ is horizontal. Then we have the horizontal covariant $(s+1)$-tensor $\Delta^{v} T$, which will be called the covariant $v$-derivative of $T$.

Lemma 3.8. 1) If $T$ is a horizontal covariant symmetric tensor on $B$, then its covariant $v$-derivative $\Delta^{\prime \prime} T$ has the property that $\Delta^{v} T\left(X_{1}, \cdots, X_{s}, Y\right), X_{1}, \cdots, X_{s}, Y \in B_{b}$, is symmetric with respect to $X_{1}, \cdots, X_{s}$.

2) Let $f$ be a real function on $B$, and then we have the horizontal covariant s-tensor $\Delta^{v} \cdots \Delta^{v} f$. This tensor is symmetric for $s \geqq 2$.

Proof. From the definition of the operator $\left.\Delta^{v}, 1\right)$ is clearly satisfied, and hence we shall prove 2) by the process of mathematical induction. We observe first that, for $X, Y \in B_{b}$,

$$
\Delta^{v} \Delta^{v} f(X, Y)=Y^{v}\left(X^{v}(f)\right)+\left[X, Y^{v}\right]^{v}(f) .
$$

In consequence of this and Lemma 3.4 , it is easily seen that $\Delta^{\prime \prime} \Delta^{\prime \prime} f$ is symmetric. Next, we assume that $\underbrace{\Delta^{v} \cdots \Delta^{v} f}_{r}$ be symmetric for $r=2, \cdots, s$. If we write $\frac{\Delta^{v} \cdots \Delta^{v}}{s-1}=T$, then, by direct calculation,
we obtain

$$
\begin{aligned}
& \underbrace{\Delta^{v} \cdots \Delta^{v}}_{s+1} f\left(Y, X_{2}, \cdots, X_{s}, X\right)-\underbrace{\Delta^{v} \cdots \Delta^{v}}_{s+1} f\left(X, X_{2}, \cdots, X_{s}, Y\right) \\
& =\left(\left[X^{v}, Y^{v}\right]+\left[Y, X^{v}\right]^{v}-\left[X, Y^{v}\right]^{v}\right)\left(T\left(X_{2}, \cdots, X_{s}\right)\right) \\
& +\sum_{i=2}^{s} T\left(X_{2}, \cdots, Z_{(i)}, \cdots, X_{s}\right),
\end{aligned}
$$

where $X, Y, X_{2}, \cdots, X_{s} \in B_{b}$, and we put

$$
\begin{aligned}
Z_{i}=\left[X_{i},\left[Y, X^{v}\right]^{v}\right]+ & {\left[\left[X_{i}, X^{v}\right], Y^{v}\right]-\left[X_{i},\left[X, Y^{v}\right]^{v}\right] } \\
- & {\left[\left[X_{i}, Y^{v}\right], X^{v}\right] . }
\end{aligned}
$$

As a result of Lemma 3.4, we see that the first term of the right hand members vanishes and that $Z_{i}$ are equal to zero by means of the Jacobi's identity for the brackets. Thus we establish the lemma.

Lemma 3.9. Let $T$ and $T^{\prime}$ be horizontal covariant tensors or functions on $B$ and $T \cdot T^{\prime}$ their product. Then we have

$$
\Delta^{v}\left(T \cdot T^{\prime}\right)=\Delta^{v} T \cdot T^{\prime}+T \cdot \Delta^{v} T^{\prime} .
$$

The proof of this lemma is quite easy. Now, the next lemma 
gives the relation between the convariant $v$-differentiation and the dual of central transformation of $B$.

Lemma 3.10. Let $T$ be a horizontal covariant tensor or a function on $B$. Then we obtain

$$
\Delta^{v}\left(C_{z}{ }^{*}(T)\right)=(z) \cdot C_{z}{ }^{*}\left(\Delta^{v} T\right),
$$

where $(z)$ is a real number corresponding to the element $z \in Z$.

Proof. We remark first that $C_{z}^{*}(T)$ is horizontal, because $C_{z}$ maps a vertical vector into a vector of the same kind. We shall prove the lemma for a horizontal covariant $s(\geqq 1)$-tensor $T$. For $X_{1}, \cdots, X_{s}, Y \in B_{b}$, we have

$$
\begin{aligned}
& \left(\Delta^{v}\left(C_{z}{ }^{*}(T)\right)\right)\left(X_{1}, \cdots, X_{s}, Y\right)=Y^{v}\left(T\left(C_{z}\left(X_{1}\right), \cdots, C_{z}\left(X_{s}\right)\right) \circ C_{z}\right) \\
& \quad+\sum_{i=1}^{s} T\left(C_{z}\left(X_{1}\right), \cdots,\left[C_{z}\left(X_{i}\right), C_{(i)}\left(Y^{v}\right)\right], \cdots, C_{z}\left(X_{s}\right)\right) .
\end{aligned}
$$

According to Lemma 3.2, this is rewritten in the form

$$
\begin{aligned}
= & (z) \cdot\left(C_{z}(Y)\right)^{v}\left(T\left(C_{z}\left(X_{1}\right), \cdots, C_{z}\left(X_{s}\right)\right)\right) \\
& +(z) \cdot \sum_{i=1}^{s} T\left(C_{z}\left(X_{1}\right), \cdots,\left[C_{z}\left(X_{i}\right),\left(C_{z}(Y)\right)^{v}\right], \cdots, C_{z}\left(X_{s}\right)\right) \\
= & (z) \cdot \Delta^{v} T\left(C_{z}\left(X_{1}\right), \cdots, C_{z}\left(X_{s}\right), C_{z}(Y)\right) .
\end{aligned}
$$

This concludes the proof of the lemma.

Lemma 3.11. 1) For a real function $f$ on $B$, we have the reduced function $\left(\Delta^{v} f\right)_{0}$ from the covariant $v$-derivative $\Delta^{v} f$ of $f$. Then we obtain $\left(\Delta^{v} f\right)_{0}(b)=\vec{b}(f), b \in B$.

2) Let $T$ be a horizontal covariant $s(\geqq 1)$-tensor on $B$, and then we have, for $X_{1}, \cdots, \hat{X}_{i}, \cdots, X_{s}, Y \in B_{b}$, and $i=1, \cdots, s$,

$$
\begin{aligned}
& \left(\Delta^{v} T\right)_{o(i)}\left(X_{1}, \cdots, \hat{X}_{i}, \cdots, X_{s}, Y\right) \\
= & \left(\Delta^{v}\left(T_{0(i)}\right)\right)\left(X_{1}, \cdots, \hat{X}_{i}, \cdots, X_{s}, Y\right)-T\left(X_{1}, \cdots, \underset{(i)}{Y}, \cdots, X_{s}\right) .
\end{aligned}
$$

Proof. Since $\left(\vec{b}^{h}\right)^{v}=\vec{b}$, we have 1) easily. We shall show 2 ).

$$
\begin{aligned}
& \left(\Delta^{v} T\right)_{0(i)}\left(X_{1}, \cdots, \hat{X}_{i}, \cdots, X_{s}, Y\right)=\Delta^{v} T\left(X, \cdots, \vec{b}_{(i)}^{h}, \cdots, X_{s}, Y\right) \\
& =Y^{v}\left(T\left(X_{1}, \cdots, \vec{b}_{(i)}^{h}, \cdots, X_{s}\right)\right)+\sum_{a=1}^{i-1} T\left(X_{1}, \cdots,\left[X_{(a)}, Y^{(i)}\right], \cdots, \vec{b}_{(i)}^{b}, \cdots, X_{s}\right) \\
& +T\left(X_{1}, \cdots,\left[\overrightarrow{b^{h}}, Y^{v}\right], \cdots, X_{s}\right) \\
& +\sum_{a=i+1}^{s} T\left(X_{1}, \cdots,{ }_{(i)}, \cdots,\left[X_{(a)}, Y^{v}\right], \cdots, X_{s}\right) .
\end{aligned}
$$

In the third term of the right hand members, we can replace $\left[\vec{b}^{h}, Y^{\nu}\right]$ by $-Y$ in virtue of Lemma 3.6 and hence 2 ) is proved. 


\section{Chapter IV. Euclidean connections in linear Finsler bundles}

\section{\$1. The fundamental function and metric tensor}

We consider a covariant $s(\geqq 0)$-tensor $T$ on $B$, and suppose that $T$ satisfy the equation

$$
C_{z}^{*}(T)=(z)^{r} \cdot T
$$

where $C_{z}$ is any central transformation of $B$. Then $T$ will be said to be $r$-homogeneous. In particular, that $T$ is 0 -homogeneous means that $T$ is central invariant. We write by $R^{+}$the set of positive numbers, and if the above equation holds for any $z,(z) \in R^{+}$ only, then $T$ will be said to be positively $r$-homogeneous. We shall define the homogenity for differentiable forms similarly. On making use of the covariant $v$-derivative, the Euler's theorem for homogeneous functions leads us to the fact that a covariant $s$-tensor $T$ is $r$-homogeneous, if and only if the equation

$$
\left(\Delta^{v} T\right)_{0(s+1)}=r \cdot T
$$

be satisfied (cf. Lemma 3. 11, 2)).

Lemma 4.1. Let $T$ be a $r$-homogeneous covariant $s(\geqq 1)$-tensor on B. Then the induced horizontal tensor $T^{h}$ is $(r-s)$-homogeneous. The same is true for a form.

Proof. From the definition of the horizontal tensor $T^{h}$, we have

$$
\begin{aligned}
& \left(C_{z}^{*}\left(T^{h}\right)\right)\left(X_{1} \cdots, X_{s}\right)=T^{h}\left(C_{z}\left(X_{1}\right), \cdots, C_{z}\left(X_{s}\right)\right) \\
& =T\left(\left(C_{z}\left(X_{1}\right)\right)^{v}, \cdots,\left(C_{z}\left(X_{s}\right)\right)^{v}\right), \quad X_{1}, \cdots, X_{s} \in B_{b} .
\end{aligned}
$$

From Lemma 3.2, we can rewrite this in the form

$$
\begin{aligned}
& =(z)^{-s} \cdot T\left(C_{z}\left(X_{1}^{v}\right), \cdots, C_{z}\left(X_{s}^{v}\right)\right)=(z)^{-s} \cdot\left(C_{z}^{*}(T)\right)\left(X_{1}^{v}, \cdots, X_{s}^{v}\right) \\
& =(z)^{r-s} \cdot T^{h}\left(X_{1}, \cdots, X_{s}\right) .
\end{aligned}
$$

This proves the lemma.

Now we consider the local expressions $\omega_{a}$ on $V_{a}$ of the Finsler connection form $\omega$. Since we have shown that $\omega_{\infty}$ is central invariant, we obtain, as a result of Lemma 4.1, the following theorem.

Theorem 4.1. Let $\omega_{\infty}$ be the local expressions on $V_{a}$ of the Finsler connection form $\omega$. The local horizontal forms $\left(\omega_{\infty}\right)^{h}$ are (-1)-homogeneous. 
The forms $\left(\omega_{o}\right)^{h}$ are called the local horizontal connection forms. Let $\omega_{\alpha}$ and $\omega_{\beta}$ be local expressions of $\omega$ on $V_{\alpha}$ and $V_{B}$ respectively. Then it is well known that we obtain the equations

$$
\omega_{\beta}=\operatorname{ad}\left(g_{\alpha \beta}^{-1}\right) \cdot \omega_{\alpha}+g_{\alpha \beta}^{-1} \cdot \delta g_{\alpha \beta},
$$

where $g_{\alpha \beta}: V_{\alpha} \cap V_{\beta} \rightarrow G$ satisfy the equations $\sigma_{\beta}=\sigma_{\alpha} \cdot g_{\alpha \beta}$ for the local sections $\sigma_{a}$ and $\sigma_{\beta}$. Since we obtain

$$
\eta \circ \sigma_{\beta}(b)=\rho_{\beta}(x)=\rho_{a}(x) \cdot g_{\alpha \beta}(b), \quad b \in V_{a} \cap V_{\beta}, x=\tau(b),
$$

the mappings $g_{\alpha \beta}$ are constant on each fibre $V(x)$, and hence the differentials $\delta g_{\alpha \beta}$ carry a vertical vector to zero. Then we see

$$
\left(\omega_{B}\right)^{h}(X)=\operatorname{ad}\left(g_{\alpha \beta}^{-1}\right) \cdot\left(\omega_{\alpha}\right)^{h}(X), \quad X \in B_{b} .
$$

This means that the local horizontal connection forms are of ad(G)type. Making use of (7), we have easily that

$$
\left(\omega_{a}\right)^{h}=C_{j k}^{i}\left(x^{1}, \cdots, x^{n}, b^{1}, \cdots, b^{n}\right) d x^{k} \cdot \hat{g}_{i}^{j} .
$$

That $\left(\omega_{\infty}\right)^{h}$ is of $a d(G)$-type means that the set of $C_{j_{k}}^{i}$ obeys the transformation of the well-known tensor-type by the transformation of canonical coordinates. And the above theorem shows that $C_{j k}^{i}$ are $(-1)$-homogeneous functions with respect to the variables $b^{1}, \cdots, b^{n}$ (cf. $\$ 7$ ).

We shall return to the consideration of general homogeneous tensors and prove that

Lemma 4.2. Let $T$ be a $r$-homogeneous covariant $s(\geqq 1)$-tensor on $B$. Then the reduced tensors $T_{0(i)}, i=1, \cdots, s$, are $(r+1)$-homogeneous. The same is true of a form.

Proof. From Lemmas 3.2 and 3.3, it follows that

$$
\left((z) \cdot C_{z}\left(\vec{b}^{h}\right)\right)^{v}=C_{z}(\vec{b})=(\overrightarrow{z \cdot b}), \quad b \in B,
$$

and hence we can choose $(z) \cdot C_{z}\left(\vec{b}^{h}\right)$ as a vector inducing the characteristic vector $(\overrightarrow{z \cdot b})$ at the point $z \cdot b$. Therefore we obtain, for $X_{2}, \cdots, X_{s} \in B_{b}$,

$$
\begin{aligned}
& \left(C_{z}^{*}\left(T_{0(1)}\right)\right)\left(X_{2}, \cdots, X_{s}\right)=T\left((\overrightarrow{z \cdot b})^{h}, C_{z}\left(X_{2}\right), \cdots, C_{z}\left(X_{s}\right)\right) \\
= & (z) \cdot T\left(C_{z}\left(\vec{b}^{h}\right), C_{z}\left(X_{2}\right), \cdots, C_{z}\left(X_{s}\right)\right)=(z)^{r+1} \cdot T\left(\vec{b}^{h}, X_{2}, \cdots, X_{s}\right) \\
= & (z)^{r+1} \cdot T_{0(1)}\left(X_{2}, \cdots, X_{s}\right) .
\end{aligned}
$$

This proves the lemma. 
Lemma 4. 3. Let $T$ be a $r$-homogeneous covariant $s(\geqq 0)$-tensor on B. Then $\underbrace{\Delta^{v} \cdots \Delta^{v}}_{u} T$ is $(r-u)$-homogeneous.

Proof. It follows from Lemma 3.9 that

$$
\Delta^{v}\left(C_{z}^{*}(T)\right)=\Delta^{v}\left((z)^{r} \cdot T\right)=(z)^{r} \cdot \Delta^{v} T .
$$

On the other hand, it follows from Lemma 3.10 that

$$
\Delta^{v}\left(C_{z}^{*}(T)\right)=(z) \cdot C_{z}^{*}\left(\Delta^{v} T\right) .
$$

Hence $\Delta^{v} T$ is $(r-1)$-homogeneous. The lemma will be established if we repeat the above process.

We are now in a position to define the fundamental function of a Finsler manifold. We suppose that we are given a positive valued function $L$ on $B$, which satisfies

1) L is positively 1-homogeneous.

If we put $F=\frac{1}{2} L^{2}$, then we see that $\Delta^{\prime \prime} \Delta^{\prime \prime} F$ is positively 0 -homogeous horizontal symmetric covariant 2 -tensor on $B$, in virtue of Lemmas $3.8,2$ ) and 4.3. Hence we can define the projection $\left(\Delta^{v} \Delta^{v} F\right)(b)$ on the base manifold $M$. We impose upon $L$ the further condition :

2) $\left(\Delta^{v} \Delta^{v} F\right)(b)$ is positive-definite, that is,

$$
\left(\Delta^{v} \Delta^{v} F\right)(b)(X, X) \geqq 0, \quad X \in M_{x}, b \in \tau^{-1}(x),
$$

and the equality holds if and only if $X=0$.

The function $L$ (or $F$ ) satisfying the above two conditions will be called the fundamental function of the Finsler bundle $\mathfrak{Q}$, and then $M$ the Finsler manifold. The value $L(b)$ of $L$ at a point $b \in B$ is called the Finslerian length of the tangent vector $b \in M_{x}, x=\tau(b)$. Since $L$ is positively 1 -homogeneous, then we obtain $L(z \cdot b)=$ $(z) \cdot L(b),(z) \in R^{+}$.

Next, we shall introduce the metric tensor. We suppose that a field of covariant 2 tensor $m$ is given on $B$, which satisfies the following conditions :

1) $m$ is horizontal and symmetric,

2) $m$ is 0-homogeneous.

3) the projection $m(b)$ on $M$ is positive-definite.

Then we shall call $m$ the metric tensor on $B$. For a tangent vector $\underline{X} \neq 0$ at $x$ of $M$, we obtain a positive number $\sqrt{m(b)(X, X)}$, $b \in \tau^{-1}(x)$, which is called the relative euclidean length of $X$ with 
respect to the element of support $b$. From the definition of the projection $m(b)$ and the condition 2) of $m$, it follows immediately that $m(z \cdot b)(\underline{X}, \underline{X})=m(b)(\underline{X}, \underline{X}), z \in Z$. We construct the reduced covariant vector $m_{0}$ from $m$, which is 1-homogeneous in virtue of Lemma 4.2. $m_{0}$ is called the characteristic form and denoted by $\stackrel{b}{\rightarrow}$ Thus we have $\underset{\rightarrow}{b}(X)=m\left(\vec{b}^{h}, X\right), X \in B_{b}$. The reduced function $\overrightarrow{b_{0}}$ from $\underset{\rightarrow}{b}$ is given by $\underset{\rightarrow}{b_{0}}(b)=m\left(\overrightarrow{b^{h}}, \overrightarrow{b^{h}}\right)=m(b)(b, b), b \in B$, from which it follows that the value of $b_{\rightarrow}$ at $b \in B$ is the square of the relative euclidean length of $b \in M_{x}, x=\tau(b)$, with respect to the element of support $b$ itself. The quantity $\sqrt{b_{0}(b)}$ is called the absolute length of the vector $b$.

\section{$\S 12$. Pure-horizontal subspaces}

We consider the associated bundle $\tilde{\mathfrak{B}}$ with the Finsler bundle $\mathfrak{Q}$, which has been looked upon as the vertical tangent bundle of $B$ as shown in $\S 8$. Hence we can define the covariant derivative $D_{Y} X$ of a vertical vector field $X$ on $B$ with respect to a vector field $Y$ on $B[7, \mathrm{p} .52]$. That is, let $C=\left\{b_{t}, 0 \leqq t \leqq 1\right\}$ be a integral curve of $Y$ issuing from $b_{0} \in B$ and $\bar{C}=\left\{q_{t}, 0 \leqq t \leqq 1\right\}$ a lift to $Q$ of $C$ issuing from $q_{0} \in \sigma^{-1}\left(b_{0}\right)$. Then $D_{Y} X$ is given by

$$
\left(D_{Y} X\right)_{b_{0}}=\lim _{t \rightarrow 0} \frac{1}{t}\left(q_{0} \circ q_{t}^{-1}\left(X_{b_{t}}\right)-X_{b_{0}}\right) .
$$

The vertical vector $X_{b_{t}}$ is expressed by a pair $\left(b_{t}, b_{t}^{\prime}\right)$ and we put $q_{t}=\left(b_{t}, p_{t}\right)$, and then we obtain

$$
q_{0} \circ q_{t}^{-1}\left(X_{b_{t}}\right)=q_{0}\left(p_{t}^{-1}\left(b_{t}^{\prime}\right)\right)=\left(b_{0}, p_{0} \circ p_{t}^{-1}\left(b_{t}^{\prime}\right)\right) .
$$

Therefore we have the expression of $D_{Y} X$ as follows:

$$
\left(D_{Y} X\right)_{b_{0}}=\left(b_{0}, \lim _{t \rightarrow 0} \frac{1}{t}\left(p_{0} \circ p_{t}^{-1}\left(b_{t}^{\prime}\right)-b_{0}^{\prime}\right)\right) \text {. }
$$

We consider a vector field $X$ on $B$, which is not necessarily to be vertical. Then we have the vertical vector field $X^{v}$, and hence we can define the convariant derivative $D_{Y} X^{v}$, which will be written in the form $D_{Y}^{p} X$.

Next, we consider a horizontal covariant $s$-tensor $T$ on $B$, and define the covariant derivative $D_{Y}^{o} T$ of $T$ with respect to $Y$ as follows $[7$, p. 55$]$ : 


$$
D_{Y}^{v} T\left(X_{1}, \cdots, X_{s}\right)=Y\left(T\left(X_{1}, \cdots, X_{s}\right)\right)-\sum_{i=1}^{s} T\left(X_{1}, \cdots,\left(D_{(i)}^{v} X_{i}\right)^{h}, \cdots, X_{s}\right),
$$

where $X_{1}, \cdots, X_{s} \in B_{b}$. The covariant differental $D^{v} T$ of $T$ is the $(s+1)$-tensor, which is given by $D^{v} T\left(X_{1}, \cdots, X_{s}, Y\right)=D_{Y}^{v} T\left(X_{1}, \cdots\right.$, $\left.X_{s}\right), X_{1}, \cdots, X_{s}, Y \in B_{b}$.

Next, let $X$ be a vector field on $M$ and $b_{t}, 0 \leqq t \leqq 1$, a vector field defined along a curve $\underline{C}=\left\{x_{t}, 0 \leqq t \leqq 1\right\}$ on $M$. The curve $C=\left\{b_{t}\right\}$ is in $B$ and covers $\underline{C}$. Let $C^{*}=\left\{p_{t}, 0 \leqq t \leqq 1\right\}$ be a life to $P$ of $C$ issuing from a point $p_{0} \in \rho^{-1}\left(x_{0}\right)$, and we define

$$
\left(D_{b} \underline{X}\right)_{x_{0}}=\lim _{t \rightarrow 0} \frac{1}{t}\left(p_{0} \circ p_{t}^{-1}\left(\underline{X}_{x_{t}}\right)-\underline{X}_{x_{0}}\right),
$$

which is clearly a tangent vector at $x_{0} . D_{b} X$ as thus defined will be called the covariant derivative of $\underline{X}$ with respect to the element of support $b_{t}$. We take a tangent vector $Y$ of the curve $C$ and a vertical vector field $X=\left(b_{t}, X\right)$. Then we have from (15) that $D_{Y} X=\left(b, D_{b} \underline{X}\right)$. Making use of $(9)$, we obtain easily

$$
D_{b} \underline{X}=\left[\frac{d X^{i}}{d t}+X^{j}\left(\Gamma_{j k}^{i}(b) \frac{d x^{k}}{d t}+C_{j k}^{i}(b) \frac{d b^{k}}{d t}\right)\right] \quad\left(\frac{\partial}{\partial x^{i}}\right)_{x},
$$

where $\left.X=X^{i}(\partial / \partial) x^{i}\right)_{x}, b=\left(x^{i}, b^{i}\right)$.

In particular, we can consider the case where the curve $\underline{C}$ is a single point $x_{0}$ and $C$ a vertical curve in the fibre $V\left(x_{0}\right)$. Then we obtain

$$
\left(D_{b} \underline{X}\right)_{x_{0}}=\lim _{t \rightarrow 0} \frac{1}{t}\left(p_{0} \circ p_{t}^{-1}(\underline{X})-\underline{X}\right), \quad \underline{X} \in M_{x_{0}},
$$

and such a covariant derivative of a fixed vector $X$ for a rotation of the element of support $b_{t}$ will be denoted by $D_{b}^{0} X$. From (16) it follows that $D_{b}^{0} X=X^{j} C_{j_{k}}^{i}(b)\left(d b^{k} / d t\right)\left(\partial / \partial x^{i}\right)_{x}$, where $\left(b^{k}(t)\right)$ is the vertical curve $C$. In terms of the local horizontal connection form $\left(\omega_{\infty}\right)^{h}$, this equation is written in the form

$$
D_{b}^{0} \underline{X}=\left(\omega_{\alpha}\right)^{h}\left(Y^{h}\right) \cdot \underline{X},
$$

where $Y$ is the vertical vector tangent to $\left(b_{t}\right)$, and the dot denotes the product of matrices $\left(\omega_{x}\right)^{h}\left(Y^{h}\right)$ and $X$.

We suppose now that the Finsler connection under consideration has the property $D$. We take a fixed point $x_{0} \in M$ and a curve $\underline{C}=\left\{x_{t}, 0 \leqq t \leqq 1\right\}$ on $M$ issuing form $x_{0}$. Then we shall show that there exists an unique curve $C=\left\{b_{t}, 0 \leqq t \leqq 1\right\}$ on $B$, 
covering $\underline{C}$ and issuing from a given point $b_{0} \in \tau^{-1}\left(x_{0}\right)$, such that the covariant derivative $D_{b} b$ of $b$ with respect to the element of support $b$ itself vanishes identically. In fact, from (11) and (16), we obtain the differential equations of such a curve $C$ as follows:

$$
\frac{d b^{i}}{d t}+b^{j} \Gamma_{j_{k}}^{i}(b) \frac{d x^{k}}{d t}=0,
$$

with the initial condition $b^{i}(0)=b_{0}^{i}$, where $\left(x^{k}(t)\right)$ expresses the curve $\underline{C}$ on $M$. Therefore the curve $C$ is uniquely determined by the curve $\underline{C}$ on $M$ and the starting point of $C$. The curve $C$ will be called the horizontal curve, covering $\underline{C}$, or the lift of $\underline{C}$ to $B$. Let $H_{b}$ be a set of tangent vectors at $b$ of $B$, which are tangent to lifts of curves on $M$ issuing from $x=\tau(b)$. We shall call $H_{b}$ the horizontal subspace of $B_{b}$. It is clear that $B_{b}$ is the direct sum of $H_{b}$ and $B_{b}^{v}$. For any $X \in M_{x}$, there is an unique horizontal vector $l_{b}(\underline{X})$ at $b \in \tau^{-1}(x)$, such that $\tau\left(l_{b}(\underline{X})\right)=\underline{X}$. The vector $l_{b}(\underline{X})$ is called the lift at $b$ of $\underline{X}$. In consequence of (18), the expression of the lift $l_{b}(\underline{X})$ at $b=\left(x^{i}, b^{i}\right)$ of a vector $\underline{X}=X^{i}\left(\partial / \partial x^{i}\right)_{x}$ is given by the equation

$$
l_{b}(\underline{X})=X^{i}\left(\frac{\partial}{\partial x^{i}}\right)_{b}-b^{j} \Gamma_{j k}^{i}(b) X^{k}\left(\frac{\partial}{\partial b^{i}}\right)_{b} .
$$

For $X \in B_{b}$, we can write uniquely $X=v(X)+h(X)$, where $v(X)$ is vertical and $h(X)$ horizontal. If we put $X=X^{i}\left(\partial / \partial x^{i}\right)_{b}+$ $X^{(i)}\left(\partial / \partial b^{i}\right)_{b}$, then $h(X)$ is given by the same equation (19), and hence $v(X)$ is given by

$$
v(X)=\left(X^{(i)}+b^{j} \Gamma_{j k}^{i}(b) X^{k}\right)\left(\frac{\partial}{\partial b^{i}}\right)_{b} .
$$

It is to be noted here that the vertical component $v(X)$ is different from the induced vertical vector $X^{v}$.

We consider a $s$ form $\alpha$ on $B$ and put

$$
\begin{aligned}
& \alpha^{0}\left(X_{1}, \cdots, X_{s}\right)=\alpha\left(v\left(X_{1}\right), \cdots, v\left(X_{s}\right)\right), \\
& \alpha^{1}\left(X_{1}, \cdots, X_{s}\right)=\alpha\left(h\left(X_{1}\right), \cdots, h\left(X_{s}\right)\right), \quad X_{1}, \cdots, X_{s} \in B_{b} .
\end{aligned}
$$

Then we obtain two forms $\alpha^{0}$ and $\alpha^{1}$, the latter being horizontal. The form $\alpha^{0}$ (resp. $\alpha^{1}$ ) is called the vertical (resp. horizontal) component of $\alpha$. Further we have the form $\alpha-\alpha^{0}-\alpha^{1}$, which is called the mixed component of $\alpha$. It is clear that the mixed component of a 1 -form is equal to zero. 
Let $\omega_{\alpha}$ be local expressions of the Finsler connection form $\omega$. The vertical and horizontal components of $\omega_{\infty}$ are given by

$$
\left(\omega_{a}\right)^{0}=\left(\Gamma_{j k}^{0 i} d x^{k}+C_{j k}^{j} d b^{k}\right) \hat{g}_{i}^{j}, \quad\left(\omega_{a}\right)^{1}=\Gamma_{j k}^{* i} d x^{k} \hat{g}_{i}^{j},
$$

where we put

$$
\Gamma_{j k}^{* i}=\Gamma_{j_{k}}^{i}-C_{j h}^{i} \Gamma_{l k}^{h} b^{l}, \quad \Gamma_{j k}^{0 i}=\Gamma_{j_{k}}^{i}-\Gamma_{j k}^{* i} .
$$

The coefficients $\Gamma_{\xi_{k}}^{* i}$ have been introduced by E. Cartan [2, p. 14] and will play a röle in the following.

We consider next the bundle space $Q$ of the Finsler bundle $\Omega$. we have defined, in $\S 3$, a zero-horizontal subspace $\Gamma_{a}^{0}$ of the horizontal subspace $\Gamma_{q}$ at $q \in Q$, which is the lift of vertical subspace $B_{b}^{v}$ of $B_{b}, b=\sigma(q)$. We have now the horizontal subspace $H_{b}$, and hence can define the subspace $\Gamma_{q}^{1}$, which is the lift of $H_{b}$. We shall call $\Gamma_{q}^{1}$ the pure-horizontal subspace of $Q_{q}$. It is clear that $\Gamma_{q}$ is the direct sum of the zero-horizontal subspace $\Gamma_{q}^{0}$ and pure-horizontal subspace $\Gamma_{q}^{1}$. Thus we obtain the unique decomposition of a vector $\bar{X} \in Q_{q}$ as follows :

$$
\bar{X}=v(\bar{X})+h^{0}(\bar{X})+h^{1}(\bar{X}), \quad h^{0}(\bar{X}) \in \mathrm{I}_{q}^{0}, h^{1}(\bar{X}) \in \mathrm{I}_{q}^{1} .
$$

If we put $\bar{X}=X^{i}\left(\partial / \partial x^{i}\right)_{q}+X^{(i)}\left(\partial / \partial b^{i}\right)_{q}+X_{j}^{i}\left(\partial / \partial p_{j}^{i}\right)_{q}$, then the purehorizontal component $h(\bar{X})$ of $\bar{X}$ is given, in virtne of (8) by the equation

$$
\begin{array}{r}
h(\bar{X})=X^{i}\left(\frac{\partial}{\partial x^{i}}\right)_{q}-b^{j} \Gamma_{j k}^{i}(\sigma(q)) X^{k}\left(\frac{\partial}{\partial b^{i}}\right)_{q} \\
-p_{j}^{k} \Gamma_{k l}^{* i}(\sigma(q)) X^{l}\left(\frac{\partial}{\partial p_{j}^{i}}\right)_{q} .
\end{array}
$$

We consider a horizontal $s$ form $\alpha$ on $Q$, and then we have two forms $\alpha^{0}$ and $\alpha^{1}$, such that, for $X_{1}, \cdots, X_{s} \in Q_{q}$,

$$
\begin{aligned}
& \alpha^{0}\left(X_{1}, \cdots, X_{s}\right)=\alpha\left(h^{0}\left(X_{1}\right), \cdots, h^{0}\left(X_{s}\right)\right), \\
& \alpha^{1}\left(X_{1}, \cdots, X_{s}\right)=\alpha\left(h^{1}\left(X_{1}\right), \cdots, h^{1}\left(X_{s}\right)\right),
\end{aligned}
$$

which will be called the zero and pure components of $\alpha$ respectively. The form $\alpha-\alpha^{0}-\alpha^{1}$ will be called the mixed component of $\alpha$. It is clear that the mixed component of a 1 -form vanishes.

We consider finally the principal bundle $\mathfrak{*}$. Given a point $p \in P$, we take an arbitrary point $b \in \tau^{-1} \circ \rho(p)$, and then we obtain a point $q=(b, p) \in Q$. We now define the subspace $H^{*}(b)_{p}$ of $P_{p}$ as $\eta\left(\Gamma_{q}^{1}\right)$, where $\eta$ is the canonical projection $Q \rightarrow P . H^{*}(b)_{p}$ is 
called the horizontal subspace of $P_{p}$ with respect to the point $b \in B$. Let $X^{*}$ be a tangent vector at $p$ and put $h_{b}\left(X^{*}\right)=\eta^{\circ} l_{q} \circ l_{b} \circ \rho\left(X^{*}\right)$. It is clear that $\rho\left(h_{b}\left(X^{*}\right)\right)=\rho\left(X^{*}\right)$, so that $X^{*}-h_{b}\left(X^{*}\right)$ is vertical. Hence $P_{p}$ is the direct sum of $H^{*}(b)_{p}$ and the vertical subspace $G(x)_{p}, x=\rho(p)$. It is to be remarked that this decomposition of $P_{p}$ will depend upon the choice of a point $b \in \tau^{-1} \circ \rho(p)$ generally. It is easily shown that, if we put $X^{*}=X^{i}\left(\partial / \partial x^{i}\right)_{p}+X_{j}^{i}\left(\partial / \partial p_{j}^{i}\right)_{p}$, then the horizontal component $h_{b}\left(X^{*}\right)$ is given by

$$
h_{b}\left(X^{*}\right)=X^{i}\left(\frac{\partial}{\partial x^{i}}\right)_{p}-p_{j}^{k} \Gamma_{k l}^{* l}(b) X^{l}\left(\frac{\partial}{\partial p_{j}^{i}}\right)_{p} .
$$

\section{$\S 13$. Torsion and curvature forms}

In this section, we suppose also that the Finsler connection under consideration has the property $D$, so that we have the notion of pure-horizontal subspaces.

Let $\theta^{*}$ be a $V$-valued 1 -form on $P$, such that $\theta_{p}^{*}\left(X^{*}\right)=$ $p^{-1} \circ \rho\left(X^{*}\right),\left[7\right.$, p. 49]. Then we have the induced form $\theta^{1}=\eta^{*}\left(\theta^{*}\right)$ on $Q$, where $\eta$ is the canonical mapping $Q \rightarrow P$, We see easily that

$$
\theta_{q}^{1}(X)=p^{-1} \circ \tau \circ \sigma(X), \quad q=(b, p), X \in Q_{q},
$$

from which it follows that $\theta^{1}$ vanishes on the zero-horizontal subspace. We shall call $\theta^{1}$ the pure-basic form on $Q$.

Next, we define a $V$-valued 1 -form $\theta^{\circ}$ on $Q$, such that

$$
\theta_{q}^{0}(X)=\delta p^{-1}(v(\sigma(X))), \quad q=(b, p), X \in Q_{q} .
$$

where $v(\sigma(X))$ is the vertical component of $\sigma(X) \in B_{b}$, and $\delta p^{-1}(v(\sigma(X)))$ is the tangent vector at $p^{-1}(b)$ of $V$, which is identified with a point of $V$. We shall call $\theta^{\circ}$ the zero-basic form on $Q$. It is clear that $\theta^{\circ}$ vanishes on the pure-horizontal subspace.

The following equations can be easily verified.

$$
\begin{gathered}
R_{g}^{\prime *}\left(\theta^{i}\right)=g^{-1} \cdot \theta^{i}, \quad i=0,1, \\
C_{z}^{\prime *}\left(\theta^{\circ}\right)=z \cdot \theta^{0}, \quad C_{z}^{\prime *}\left(\theta^{1}\right)=\theta^{1} .
\end{gathered}
$$

Thus the zero-basic form is not central invariant.

We shall now introduce the basic vector fields on $Q[7, \mathrm{p} .49]$. The pure-basic vector field $B^{1}(v)$ corresponding to an element $v \in V$ is that the value of $B^{1}(v)$ at $q \in Q$ is given by

$$
B^{1}(v)_{q}=l_{q} \circ l_{b} \circ p(v), \quad q=(b, p),
$$


where $l_{b}$ (res. $l_{q}$ ) is the operation of taking a life to $B$ (resp. Q) of a vector on $M$ (resp. $B$ ). The zero-basic vector field $B^{\circ}(v)$ corresponding to $v \in V$ is given by

$$
B^{\circ}(v)_{q}=l_{q} \circ \delta p(v), \quad q=(b, p),
$$

where $v \in V$ is considered as the tangent vector at $p^{-1}(b)$. The process which was used in $[7, \mathrm{p} .49]$ can be applied to our $B^{i}(v)$, $i=0,1$, and then we see the zero- (resp. pure-) basic vector field $X$ is a horizontal vector field on $Q$ such that $\theta^{\circ}(X)$ (resp. $\theta^{1}(X)$ ) is constant. Making use of this fact, we can easily prove the following equations.

$$
\begin{gathered}
R_{g}^{\prime}\left(B^{i}(v)\right)=B^{i}\left(g^{-1} \cdot v\right), \quad i=0,1, \\
C_{z}^{\prime}\left(B^{0}(v)\right)=B^{0}(z \cdot v), \quad C_{z}^{\prime}\left(B^{1}(v)\right)=B^{1}(v) .
\end{gathered}
$$

Thus the zero-basic vector field is not central invariant. By means of the above equations, we obtain $[7$, p. 50]

$$
\left[A, B^{i}(v)\right]=B^{i}(\hat{A} \cdot v), \quad i=0,1,
$$

where $A$ is the fundamental vector field corresponding to $\hat{A} \in \hat{G}$.

We consider the covariant differential $D \theta^{i}=\Theta i, i=0,1$, of $\theta^{i} . \quad \Theta^{0}$ (resp. $\Theta^{1}$ ) will be called the zero (resp. pure) torsion form. Then we have the pure, zero and mixed components of $\Theta^{i}$, which are written by $\Theta^{i(1)}, \Theta^{i(0)}$ and $\Theta^{i(01)}$ respectively. It is easily seen that the zero-component of $\Theta^{1}$ is equal to zero, and hence we have

$$
\begin{aligned}
& \Theta^{0}=\Theta^{0(0)}+\Theta^{0(01)}+\Theta^{0(1)}, \\
& \Theta^{1}=\Theta^{1(1)}+\Theta^{1001)} .
\end{aligned}
$$

The pure component $\Theta^{1(1)}$ of the pure torsion form $\Theta^{1}$ will be important in the final section. In terms of canonical coordinates, the local expressions $\Theta_{\alpha}^{1(1)}$ are given in the form

$$
\Theta_{\alpha}^{1(1)}=\frac{1}{2}\left(\Gamma_{j k}^{* i}-\Gamma_{k j}^{* j}\right) d x^{k} \wedge d x^{j} \cdot e_{i},
$$

where $\left(e_{i}\right)$ is a fixed base of $V$. Since E. Cartan assumed that the pure component $\Theta^{1(1)}$ vanish, then he treated only the mixed component $\Theta^{1(01)}$ of $\Theta^{1}$ and called it the torsion from [2, p. 33]. On the other hand the zero-torsion form $\Theta^{\circ}$ is not central invariant.

The additional structure equations [7, p. 51] are also obtained for $\Theta^{0}$ and $\Theta^{1}$, which are immediately given in the following. 


$$
d \theta^{i}(X, Y)=\frac{1}{2}\left(\omega(Y) \cdot \theta^{i}(X)-\omega(X) \cdot \theta^{i}(Y)\right)+\Theta^{i}(X, Y),
$$

where $i=0,1$ and $X, Y \in Q_{q}$.

We consider finally the curvature form $\Omega=D \omega$, and then we obtain the pure, zero and mixed components of $\Omega$. E. Cartan introduced the curvature forms of three kinds [2, p. 33]. In our treatment, we can obtain these forms by means of the notion of pure- and zero-horizontal subspace of $Q_{q}$. The curvature form $\Omega$ is, of course, central invariant.

\section{§14. The euclidean connection defined by E. Cartan}

E. Cartan introduced the elegant process in order to determine an euclidean connection by the fundamental function. In the final section of this paper, we shall discuss his five postulates.

We have defined, in $\S 11$, the fundamental function $L$ and the metric tensor $m$. We give now a relation between $L$ and $m$ as follows :

Postulate I. The Finsler length $L(b)$ and the absolute euclidean length $\sqrt{m(b)(b, b)}$ of any tangent vector $b \in M_{x}, x=\tau(b)$, coincide.

In terms of the function $F=\frac{1}{2} L^{2}$ and the reduced function $\underset{\rightarrow}{b}$ of the characteristic form $\underset{\rightarrow}{\rightarrow}$, the above posturate is expressed by $2 F=\underset{\rightarrow}{\rightarrow}$ at each point of $B$. Taking the covariant $v$-derivative of the both sides of this equation, we have

$$
2 \Delta^{v} F(X)=X^{v}\left(m\left(\vec{b}^{h}, \vec{b}^{h}\right)\right), \quad X \in B_{b} .
$$

Taking account of the symmetry of $m$, we have

$$
\Delta^{v} m\left(\vec{b}^{h}, \vec{b}^{h}, X\right)=X^{v}\left(m\left(\vec{b}^{h}, \vec{b}^{h}\right)\right)+2 m\left(\left[\vec{b}^{h}, X^{v}\right], \vec{b}^{h}\right),
$$

and, according to Lemma 3.6 , we obtain

$$
=X^{v}\left(m\left(\vec{b}^{h}, \vec{b}^{h}\right)\right)-2 m\left(X, \vec{b}^{h}\right) .
$$

Hence, under the above postulate, we have

$$
2 \Delta^{v} F=\left(\Delta^{v} m\right)_{0(1)(2)}+\underset{\rightarrow}{2 b} .
$$

Since $m$ is 0 -homogeneous, we obtain $\left(\Delta^{v} m\right)_{0(3)}=0$ in virtue of the Euler's theorem, while $\left(\Delta^{v} m\right)_{o(1)}$ is equal to $\left(\Delta^{v} m\right)_{0(2)}$. These are 
written by $\left(\Delta^{v} m\right)_{0}$ simply. We give now the second postulate as follows :

Postulate II. The metric tensor $m$ satisfies the condition of normality: $\left(\Delta^{v} m\right)_{0}=0[4$, p. 372$]$.

When this postulate is satisfied, the equation (23) is reduced to the simple form

$$
\Delta^{\prime \prime} F=\underset{\rightarrow}{b} .
$$

It follows from Lemma 3. 11, 2) that, for $X, Y \in B_{b}$,

$$
\Delta^{v} b(X, Y)=\left(\Delta^{v}\left(m_{0}\right)\right)(X, Y)=\left(\Delta^{v} m\right)_{0}(X, Y)+m(X, Y),
$$

and hence Postulate II and (24) give

$$
\Delta^{v} \Delta^{v} F=m \text {. }
$$

Therefore, the metric tensor $m$ is determined by the fundamental function $F$ under Postulate I and II. It is easily seen that the properties 1 ), 2) and 3) imposed upon $m$ are satisfied by means of the properties of $L$ and covariant $v$-differentiation.

The following postulate is the same as Postulate $C$ of $E$. Cartan.

Postulate III. The covariant derivative $D_{b}^{0} b^{\prime}$ of a fixed vector $b^{\prime} \in M_{x}$ for a rotation of the element of support $b$ satisfies the equation $m(b)\left(D_{b}^{0} b^{\prime}, b^{\prime \prime}\right)=m(b)\left(b^{\prime}, D_{b}^{0} b^{\prime \prime}\right)$.

In the following we shall use letters of matrices. It follows from (17) that the left hand side of the above equation is written in the form ${ }^{t} b^{\prime \prime} \cdot\left(m\left(\omega_{o}\right)^{h}\left(Y^{h}\right)\right) \cdot b^{\prime}{ }^{4)}$ Since $m$ is symmetric, Postulate III is expressible in the form

$$
{ }^{t}\left(\omega_{\infty}\right)^{h} m=m\left(\omega_{\infty}\right)^{h} .
$$

In terms of canonical coordinates, (26) is written in the wellknown form

$$
C_{j_{i k}}=C_{i j_{k}}, \quad i, j, k=1, \cdots, n, \quad \text { where } C_{i j_{k}}=g_{j l} C_{i_{k}}^{l} .
$$

In order to give the following postulate, we consider the covariant derivative $D^{v} m$ of $m$ :

$$
D^{\prime \prime} m(X, Y, Z)=Z(m(X, Y))-m\left(\left(D_{Z}^{v} X\right)^{h}, Y\right)-m\left(X,\left(D_{Z}^{v} Y\right)^{h}\right),
$$

where $X, Y, Z \in B_{b}$. In making use of canonical coordinates and taking account of (26), we obtain, from the above equation

4) The sign $t$ on the left shoulder of a matrix indicates its transposed matrix. 


$$
\left(D^{\prime \prime} m\right)^{h}=(d m)^{h}-2 m\left(\omega_{a}\right)^{h} .
$$

On the other hand, we shall write $\Delta_{Z}^{v} m(X, Y)=\Delta^{v} m(X, Y, Z)$, for $X, Y, Z \in B_{b}$. Then we obtain

$$
\begin{aligned}
\Delta_{Z}^{v} m(X, Y) & =Z^{v}(m(X, Y))+m\left(X Z^{v}-Z^{v} X, Y\right)+m\left(X, Y Z^{v}-Z^{v} Y\right) \\
& =Z^{v}\left({ }^{t} X m Y\right)-m\left(Z^{v} X, Y\right)-m\left(X, Z^{v} Y\right) \\
& ={ }^{t} X Z^{v} m Y=Z^{v}(m)(X, Y)=(d m)^{h}(Z)(X, Y) .
\end{aligned}
$$

Hence we have

$$
\Delta_{Z}^{v} m=(d m)^{h}(Z) .
$$

Now we require the following relation between the Finsler connection and the metric tensor $m$.

Postulate IV. The linear Finsler connection is metrical: $D^{n} m=0$.

In a Riemann manifold, the linear connection is determined by the similar postulate. In our case, from (27) it follows first that $(d m)^{h}=2 m\left(\omega_{\infty}\right)^{h}$. Further, in virtue of (28), we obtain $\Delta^{v} m=2 m\left(\omega_{\infty}\right)^{h}$. We have had the expression (25) of $m$, from which we obtain by covariant $v$-differentiation that

$$
\Delta^{v} \Delta^{v} \Delta^{n} F=2 m\left(\omega_{\infty}\right)^{h} .
$$

In terms of canonical coordinates, (29) is written in the wellknown form

$$
\frac{1}{2} \frac{\partial^{3} F}{\partial b^{i} \partial b^{j} \partial b^{k}}=C_{i j_{k}}, \quad i, j, k=1, \cdots, n .
$$

Consequently, the local horizontal connection forms $\left(\omega_{o}\right)^{h}$ are determined by the fundamental function $F$ under the above four postulates. The coefficients $C_{j k}^{i}$ as thus obtained satisfy the equations (11) and (12). Hence we have

Theorem 4.2. Let $\mathrm{I}$ be the Finsler connection satisfying the above four postulates. Then we obtain

1) $\Gamma$ has the property $D$.

2) The reduced functions $\left(\left(\omega_{\infty}\right)^{h}\right)_{0}$ of the local horizontal connection forms vanish identically.

We had the definition of the Finslerian length of a tangent vector on $M$, which coincides with the absolute length, in consequence of Postulate I. Therefore we can define the length of a 
vector. We consider a curve $\underline{C}=\left\{x_{t}, 0 \leqq t \leqq 1\right\}$ on $M$ and a curve $C=\left\{b_{t}, 0 \leqq t \leqq 1\right\}$ which is a field of tangent vectors of $\underline{C}$. Let $C^{\prime}=\left\{b_{t}^{\prime}, 0 \leqq t \leqq 1\right\}$ be parallel to $C$. The vector $b_{t}^{\prime}$ depends generally upon the choice of $C$. Under the above postulates, this parallel displacement is independent of the choice of $C$, provided that $C$ is a field of tangent vectors of $\underline{C}$, because the condition of Theorem 3.2 is satisfied. Hence we have the notion of the parallel displacement of a vector $b_{0}^{\prime}$ along $\underline{C}$, when $C$ is a field of tangent vectors of $\underline{C}$. Furthermore, we may define a geodesic on $M$ in the ordinary manner [4].

Finally, we consider the postulate $E$ of E. Cartan, which is expressed as follows [11, p. 68]:

"The coefficients $\Gamma_{j k}^{* i}$ which appear in the covariant differential when the displacement is such that the element of support is transported parallel to itself from $x$ to $x+d x$ are to be symmetric in their lower indices."

The coefficients $\Gamma_{j k}^{* i}$ as above mentioned coincide with the one as defined in $\$ 12$, and we can treate the pure component of the pure torsion form. Then, in our case, the above postulate is expressible as follows:

Postulate V. The pure component $\Theta^{1(1)}$ of the pure torsion form $\Theta^{1}$ vanishes identically.

Therefore $\Theta^{1}$ is equal to its mixed component $\Theta^{1(01)}$. From the definition of $\theta^{1}$, we have, for $X, Y \in Q_{q}$,

$$
\Theta^{1(1)}(X, Y)=d \theta^{*}\left(\eta\left(h^{1}(X)\right), \eta\left(h^{1}(Y)\right)\right) .
$$

Hence Postulate $\mathrm{V}$ means in $\mathfrak{s}_{s}$ that $d \theta^{*}$ vanishes on the horizontal subspace $H^{*}(b)_{p}$ of $P_{p}$ with respect to a point $b \in B$.

Under the above five postulates, we may determine uniquely the Finsler connection, and the calculation in order to show this is well-known [2], [11].

\section{BIBLIOGRAPHY}

[1] L. Auslander: On curvature in Finsler geometry, Trans. Amer. Math. Soc., 79 (1955), 378-388.

[2] E. Cartan: Les espaces de Finsler. Actualités 79 (1934)

[3] S.S. Chern: On the euclidean connections in a Finsler space, Proc. Nat. Acad. Sci. U.S.A., 29 (1943), 33-37.

[4] M. Hashiguchi: On parallel displacements in Finsler spaces, J. Math. Soc. Japan, 10 (1958), 365-379. 
[5] S. Kashiwabara: On the euclidean connections in a Finsler manifold, Tôhoku Math. J., (2), 10 (1958), 69-80.

[6] A. Lichnerowicz: Théorie globale des connexions et des groupes d'holonomie, Edizioni Cremonese, Roma, 1955.

[7] K. Nomizu: Lie groups and differential geometry, Math. Soc. Japan, 1956.

[8] T. Ōtsuki: Geodesic coordinates in Finsler spaces, Math. J. Okayama Univ., 6 (1957), 135-145.

[9] _...: Theory of affine connections of the space of tangent directions of a differentiable manifold I, II, III, ibid. 7 (1957), 1-49, 50-74, 95-122.

[10] _ - Not on curvature of Finsler manifolds, ibid. 8 (1958), 107-116.

[11] H. Rund: The differential geometry of Finsler spaces, Springer, Berlin, 1959.

[12] N. Steenrod: The topology of fibre bundles, Princeton Univ. Press, 1951. 